\title{
Impaired Mitochondrial Mobility in Charcot-Marie-Tooth Disease
}

Cara R. Schiavon ${ }^{1,2}$ and Uri Manor ${ }^{1, *}$

${ }^{1}$ Waitt Advanced Biophotonics Center, Salk Institute for Biological Studies, La Jolla, CA, USA ${ }^{2}$ Molecular and Cell Biology Laboratory, Salk Institute for Biological Studies, La Jolla, CA, USA *Corresponding author

Keywords: Organelle transport, Charcot-Marie-Tooth, Axonal transport deficiency, Neurodegeneration, Cytoskeleton, Mitochondria 


\section{Abstract}

Charcot-Marie-Tooth (CMT) disease is the most commonly inherited neurological disorder, defined by progressive deterioration of the peripheral nerves. Clinical manifestations of CMT mutations are typically limited to peripheral neurons, the longest cells in the body. Currently, mutations in at least 80 different genes are associated with CMT and new mutations are regularly being discovered. A large portion of the proteins mutated in axonal CMT have documented roles in mitochondrial mobility. This suggests that trafficking defects may be a common underlying mechanism in CMT. This review will focus on the potential role of altered mitochondrial mobility in the pathogenesis of axonal CMT, highlighting the challenges and opportunities to this "impaired mobility" model of the disease.

\section{Introduction}

Charcot-Marie-Tooth (CMT) disease is the most commonly inherited neurological disorder, affecting 1 in 2500 people (Skre, 1974). It is a peripheral neuropathy defined by progressive deterioration of the peripheral nerves in the distal parts of the body, specifically the feet, hands, and lower extremities. This typically results in both motor and sensory loss in the affected areas. Unraveling the pathomechanism(s) behind CMT is somewhat complicated by the fact that CMT is both genetically and clinically heterogeneous. CMT has many subtypes including X-linked, demyelinating (affecting mainly Schwann cells), axonal, and intermediate (affecting both axons and Schwann cells). For this review, we are focusing on axonal defects due to perturbations of mitochondrial mobility.

CMT variants were originally classified based purely on clinical data. However, a recent explosion in genetic data can be mined to generate some compelling hypotheses. To date, over 100 mutations across more than 40 different proteins have been implicated in axonal 
and intermediate CMT. A large fraction of CMT-associated proteins are shown or predicted to affect the mobility of mitochondria or other organelles (Table 1). In this review, we will focus on the role of CMT mutations in mitochondrial mobility, and attempt to lay out an "impaired mobility" hypothesis as a "grand unified theory" for why CMT mutations only affect peripheral neurons, while at the same time highlighting what we believe to be important limitations to this model. Our belief is the insights gained from studying the effects of CMT mutations in peripheral neurons will lend insight towards the role of mitochondrial mobility in other types of neurons and neurodegenerative disorders, including aging.

\section{CMT is a progressive disorder that affects only the longest neurons}

CMT affects only the distal ends of the body - the feet, hands, and lower extremities. These axons can be as long as a meter in some individuals. Some CMT mutations also cause optic atrophy, and the optical nerve notably consists of relatively long axons ( $\sim 50 \mathrm{~mm})$. Patients are usually born normal, but typically by age 10 have displayed major loss of function. That said, the range of age of onset can be very broad, ranging from toddler years to the 5th decade of life. The severity of the disease is directly correlated with the age of onset (Chung et al., 2006; Verhoeven, Claeys, Zuchner, et al., 2006), and the longer axons (i.e. the feet) invariably degenerate before the shorter axons (i.e. the hands). In cases where CMT patients also display optic atrophy, this occurs after loss of function in the hands (Verhoeven, Claeys, Zuchner, et al., 2006; Zuchner et al., 2006). Together, these observations indicate a disease that directly correlates the length of the axon with the speed of onset, and the speed of onset with the magnitude of the pathology.

\section{CMT mutations largely affect mitochondrial mobility}

As mentioned above, a unique peripheral nerve characteristic is their extreme length, which suggests these cells are uniquely sensitive to impaired long-distance transport. When considering the specific physiopathology of CMT mutations affecting only the longest axons in the body, this 
makes sense: Any reduction in mobility will first affect the cells with the most extreme demand for mobility. Put simply, a decrease in mobility would have a greater impact on longer distance commutes than shorter ones. In support of this theory, 22 out of the 47 proteins mutated in axonal or intermediate CMT can be argued to play roles in mitochondrial mobility (Table 1).

The majority of CMT studies have centered on Mitofusin 2 (MFN2) mutations, which consistently result in reduced axonal mitochondrial mobility. This has now been reproduced in mouse models and patient cell lines and tissues. Neurons expressing MFN2 CMT mutants and neurons from MFN2 CMT mouse models also show reduced axonal mitochondrial mobility (Baloh, Schmidt, Pestronk, \& Milbrandt, 2007; Rocha et al., 2018; Vallat et al., 2008). Confoundingly, MFN2 is also implicated in mitochondrial fusion dynamics, and MFN2 CMT mutations cause clustering of improperly fused mitochondria (Baloh et al., 2007; Detmer \& Chan, 2007; Rocha et al., 2018; Vallat et al., 2008). It is possible that this mitochondrial clustering contributes to reduced mitochondrial mobility. MFN2 is also implicated in mediating ER-mitochondria contacts - while MFN2 CMT mutants reduce ER-mitochondria contacts (Bernard-Marissal et al., 2019; Larrea et al., 2019), it is unclear whether these changes affect mitochondrial mobility.

However, there is clear evidence for a role in organelle-organelle contacts affecting mitochondrial mobility in CMT caused by mutations in the endo-lysosomal protein RAB7A. Wong et al. demonstrated reduced mitochondrial mobility due to prolonged inter-mitochondrial contacts in HeLa cells expressing CMT-mutant MFN2, RAB7A, or TRPV4 (Wong, Peng, \& Krainc, 2019). RAB7A CMT mutations also increase tethering between mitochondria and endolysosomes, leading to changes in mitochondrial morphology and reduced mitochondrial mobility (Cioni et al., 2019; Wong et al., 2019; Wong, Ysselstein, \& Krainc, 2018). Together, these findings are striking in that superficial interpretations of RAB7A mutations causing CMT likely focus solely on endolysosomal function. That mitochondria-organelle contacts can affect mitochondrial mobility and dynamics highlights the limitations of evaluating protein and organelle dysfunction in isolation. 
Recently, a screen for RAB7A binding partners found that another CMT protein, INF2, is one of several actin-binding candidate interaction partners for RAB7A (Pan et al., 2020). This is particularly relevant to our discussion on CMT, inter-organelle contacts, and mitochondrial mobility for multiple reasons. First, a splice isoform of INF2 is tail-anchored to the endoplasmic reticulum (ER). Second, dominant active mutations in ER-anchored INF2 that mimic INF2 CMT mutations have been shown to increase actin-dependent mitochondrial fragmentation and decrease mitochondrial mobility (Chakrabarti et al., 2018; Korobova, Ramabhadran, \& Higgs, 2013). Together, these data point towards an important role in ER-mitochondria inter-organelle contacts in somehow regulating mitochondrial mobility via the actin cytoskeleton. That INF2 also potentially interacts with RAB7A suggests that mitochondria, endo-lysosomes, and ER all contact one another via CMT-associated proteins.

All INF2 CMT mutations are predicted or shown to increase actin assembly (Bayraktar et al., 2020). While some actin-binding motor proteins likely facilitate microtubule-independent mitochondrial transport, numerous studies have shown that long-range microtubule-based mobility of mitochondria is antagonized by actin and actin-binding motor proteins (CardanhoRamos, Faria-Pereira, \& Morais, 2020; Chada \& Hollenbeck, 2004; Pathak, Sepp, \& Hollenbeck, 2010; Quintero et al., 2009; Venkatesh, Mathew, \& Koushika, 2019). Thus, while the effects of INF2 CMT mutations have yet to be studied in neurons, it is reasonable to expect that INF2 CMT mutations will cause an actin-dependent decrease in mitochondrial mobility in axons. Furthermore, since the ER regularly contacts many other organelles, and even appears to drive actin-assembly at ER-organelle contact sites (Chakrabarti et al., 2018; Korobova, Gauvin, \& Higgs, 2014; Korobova et al., 2013; Manor et al., 2015; Schiavon et al., 2020; Yang \& Svitkina, 2019), it is quite 
possible INF2 CMT mutations cause aberrant actin assembly on other organelles, reducing their mobility as well (Figure 1).

Together with the newly uncovered role for RAB7A in (indirectly) modulating actin assembly (Pan et al., 2020), these observations point towards a role for multiple CMT mutations causing aberrant organelle-organelle and organelle-actin contacts, all of which cause decreased mitochondrial mobility. Whether some (or all) CMT mutations also cause decreased mobility of other organelles remains an important open question.

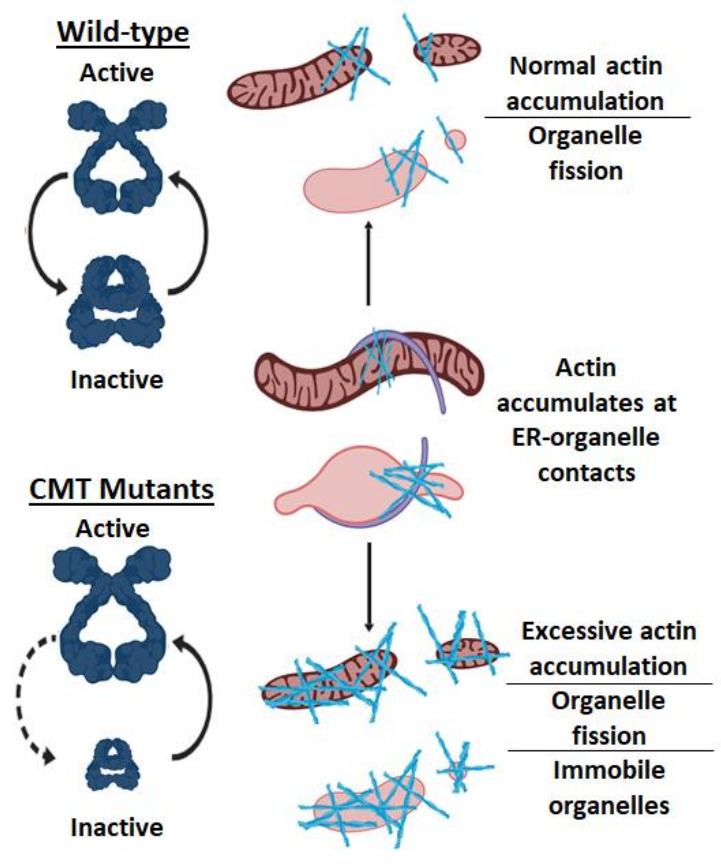

Figure 1: Model for how CMT mutations in INF2 reduce mitochondrial mobility. Normal INF2 is autoinhibited and thus has regulated actin-assembly activity. CMT mutations in INF2 reduce autoinhibition, resulting in excessive actin assembly on mitochondria and potentially other organelles, which in turn reduces their mobility.

Usually the focus of mitochondria's role in CMT has been primarily on MFN2 and GDAP1, with a lesser focus on motor proteins (KIF1B, KIF5A, DYNC1H1, DCTN2) and some cytoskeletal proteins (NEFL). Here we have introduced INF2 and RAB7A as CMT-associated proteins likely involved in mitochondrial mobility and dynamics. However, the proteins mutated in CMT that play roles in mitochondrial function (particularly mitochondrial dynamics) extend beyond those listed above (see Table 1 for a full list).

\section{Why do mobility defects only affect peripheral neurons in CMT patients?}

Hopefully we have convinced the reader that many CMT mutations likely reduce mitochondrial mobility. Given the extreme lengths of peripheral axons, it is tempting to conclude that a reduction in mobility due to CMT mutations simply affects longer axons more severely (the 
"impaired mobility model" of CMT). One can easily reconcile two key features of CMT using the impaired mobility model:

1. The progressive nature of the disease: This suggests that damage must be accumulating over time. One can imagine that damage accumulates most severely in longer axons, due to reduced turnover of damaged mitochondria resulting from reduced mitochondrial mobility. Interestingly, one could imagine that reduced mobility of other organelles associated with turnover (e.g. lysosomes) could also cause increased accumulation of damaged mitochondria in longer axons.

\section{The longest peripheral axons (i.e. the feet) progressively degenerate prior to the} next-longest axons (i.e. the hands): This further supports the impaired mobility model, wherein damage accumulates first in the longest axons due to the more demanding, "longer commute" resulting in faster accumulation of damaged mitochondria.

Unfortunately, while this model is compelling, it appears to be overly simplistic. The weakness in relying on mobility alone as an explanation can best be highlighted by comparing the lengths of different axons both within and between species. For example, the longest axon in mice is approximately $2 \mathrm{~cm}$, whereas in humans the longest axon is $\sim 60$ times longer. Just as striking, some unaffected axons in the human CNS may be longer than the mouse's longest axon. The very same mutation in humans and mice can cause CMT, yet no defects are found in the brains of human CMT patients. Meanwhile, the motor proteins, cytoskeletal tracks, and mitochondria of mice and humans are all roughly the same size, and all possess roughly the same biophysical properties (e.g. velocity, force generation, etc.) when transporting their organelles across long distances (Figure 2). 


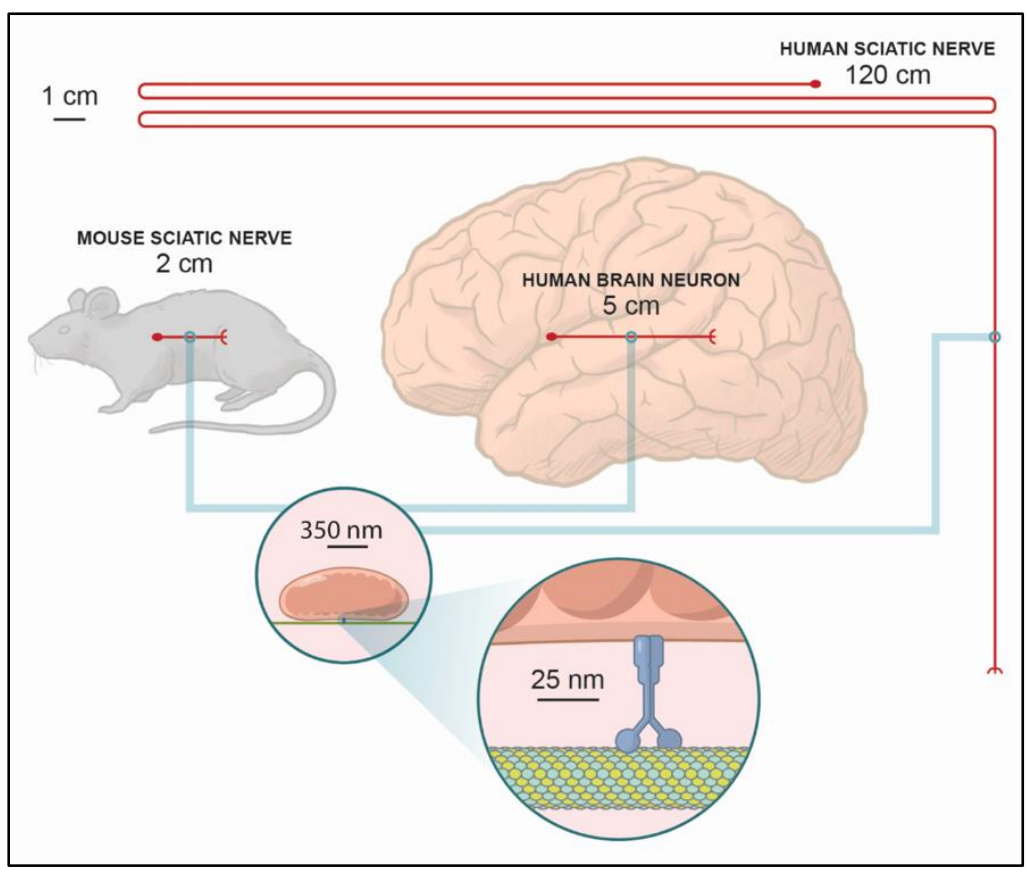

Figure 2: The relative scales of axons in humans versus mice. While microtubules, motor proteins, and mitochondria have nearly identical sizes in mice and men, the length of the axons can be very different. At the same time, axons in the mouse sciatic nerve are not as long as some of the longer axons within the human brain. For example, it is known in the macaque there are direct connections from the frontal eye fields in the anterior bank of the arcuate sulcus to the primary visual cortex, which in the macaque is $\sim 5 \mathrm{~cm}$, and probably as long as $12.5 \mathrm{~cm}$ in humans. Notably, for most CMT patients the pathology is often constrained to only peripheral neurons. Since the same mutations can cause CMT in mice and men, the distances mitochondria must travel can only provide a partial explanation for the physiopathology of these mutations.

Any analysis of mobility must consider not just distance but also time. A laboratory mouse only lives for $\sim 1-2$ years at most, while humans may not even experience symptoms until adolescence or adulthood. Perhaps the difference in relative magnitude of both time and space can perhaps at least partially be explained by interspecies differences in metabolism and oxidative stress? There is clearly a "missing variable" that also likely underlies differences in lifespan between species. Thus, CMT may serve as a "model disease" to better understand age-related neurodegeneration. It is well established mitochondria play myriad roles at the presynapse, including calcium signing, ATP production, intra- and intercellular signaling, and the biosynthesis of signaling molecules (Devine \& Kittler, 2018). Perhaps perturbed transport of mitochondria to the pre-synapse of peripheral neurons in CMT provides an opportunity to better understand other neurodegenerative disorders associated with defective presynaptic mitochondria, including Alzheimer's, ALS, Parkinson's, Friedreich's ataxia, and hereditary spastic paraplegia (Devine \& Kittler, 2018). 
But even considering the impaired mobility model for CMT within a single organism has some issues. It is difficult to imagine how reductions in mobility as high as 1000\% (Baloh et al., 2007; Rocha et al., 2018) could have a severe effect on only the longest axons, but not other axons that, while shorter than the peripheral neurons, are still very long compared to the $\sim 25 \mathrm{~nm}$ step size of a motor protein. It is not obvious that a truck unable to survive regular trips from California to Washington, DC will be perfectly fine for routes between California and Seattle, Washington.

When considering these conundrums, it is helpful to consider alternative mechanisms for replenishing mitochondria in neurons, many of which are reviewed elsewhere (Misgeld \& Schwarz, 2017; Yu \& Pekkurnaz, 2018). Briefly, mitochondrial rejuvenation is speculated to be at least partially mediated via local translation in the axon. Interestingly, multiple CMT mutations affect local translation machinery (Table 1). More recent work showed that mitochondria serve as a stable compartment for mediating biogenesis, in particular at synapses (Rangaraju, Lauterbach, \& Schuman, 2019). This raises a chicken vs. egg question: Does reduced mitochondrial mobility impair local translation needed for synaptic and therefore neuronal health and maintenance? Or does impaired local translation lead to damaged mitochondria that cannot be replaced without sufficient mobility? That CMT is caused by mutations disrupting both mobility and local translation indicates these two processes are uniquely related in long axons.

\section{Conclusion and open questions}

One open question is how the overall distribution of mitochondria is altered in CMT neurons, and how that relates to axonal maintenance. A recent study showed mitochondria tend to distribute along the length of axons with regular spacing, and that inter-mitochondrial feedback mediates their positioning and movement. Is this feedback-based positioning altered in CMT? Do mutations affecting mobility result in CMT via a "domino effect" caused by defects in 
relatively local repositioning between axonal mitochondria, which then cascades with increasing defects as a function of increasing axonal length? How much longer does it take mitochondria in CMT patients to traverse the entire length of an axon? Is there a reduction in the turnover rate of mitochondria in CMT patients? How do any and all of these factors affect mitochondria at the pre-synapse of CMT peripheral neurons, likely the most important subpopulation of mitochondria in these cells? These are surprisingly open questions we expect to be addressed in the coming years using animal and cell models of CMT.

Table 1: Genes mutated in axonal and intermediate CMT. Genes that play a role in organelle trafficking and/or mitochondrial function are marked with an asterisk.

\begin{tabular}{|l|l|l|}
\hline Gene/CMT subtype & Function & References \\
\hline AARS1/CMT2N & $\begin{array}{l}\text { Catalyzes the attachment of } \\
\text { alanine to tRNA. }\end{array}$ & $\begin{array}{l}\text { (Bansagi et al., 2015; Dohrn } \\
\text { et al., 2017; McLaughlin et } \\
\text { al., 2012; Weterman et al., } \\
\text { 2018; Z. Zhao et al., 2012) }\end{array}$ \\
\hline ATP1A1/CMT2DD & $\begin{array}{l}\text { Catalyzes the hydrolysis of ATP } \\
\text { coupled with the exchange of } \\
\text { sodium and potassium ions } \\
\text { across the plasma membrane. }\end{array}$ & (Lassuthova et al., 2018) \\
\hline BAG3/CMT2* & $\begin{array}{l}\text { Acts as a nucleotide-exchange } \\
\text { factor promoting the release of } \\
\text { ADP from the HSP70 and HSC70 } \\
\text { proteins thereby triggering } \\
\text { client/substrate protein release. } \\
\text { Has anti-apoptotic activity. Plays } \\
\text { a role in cytoskeletal proteostasis } \\
\text { and dynamics. }\end{array}$ & $\begin{array}{l}\text { (S. J. Kim et al., 2018; Shy } \\
\text { et al., 2018) }\end{array}$ \\
\hline BSCL2/CMT2* & $\begin{array}{l}\text { Plays a crucial role in the } \\
\text { formation of lipid droplets. } \\
\text { Mediates the formation and/or } \\
\text { stabilization of endoplasmic } \\
\text { reticulum-lipid droplet contacts. } \\
\text { Binds anionic phospholipids } \\
\text { including phosphatidic acid. }\end{array}$ & (Chaudhry et al., 2013) \\
\hline & $\begin{array}{l}\text { (C) } \\
\text { (C) }\end{array}$ & \\
\hline
\end{tabular}




\begin{tabular}{|c|c|c|}
\hline DCTN2/CMT2* & $\begin{array}{l}\text { Component of a large } \\
\text { macromolecular complex } \\
\text { required for the cytoplasmic } \\
\text { dynein-driven movement of } \\
\text { organelles along microtubules. } \\
\text { Pays a role in prometaphase } \\
\text { chromosome alignment and } \\
\text { spindle organization during } \\
\text { mitosis. }\end{array}$ & (Braathen et al., 2016) \\
\hline DGAT2/CMT2 & $\begin{array}{l}\text { Required for synthesis and } \\
\text { storage of intracellular } \\
\text { triglycerides. }\end{array}$ & (Hong et al., 2016) \\
\hline DHTKD1/CMT2Q & $\begin{array}{l}\text { Catalyzes the overall conversion } \\
\text { of } 2 \text {-oxoglutarate to succinyl-CoA } \\
\text { and } \mathrm{CO}_{2} \text {. }\end{array}$ & $\begin{array}{l}\text { (Baets, De Jonghe, \& } \\
\text { Timmerman, 2014; Dohrn et } \\
\text { al., 2017; Z. H. Zhao, Chen, } \\
\text { Zhou, \& Wang, 2019) }\end{array}$ \\
\hline DNAJB2/CMT2T & $\begin{array}{l}\text { Functions as a co-chaperone, } \\
\text { activating the ATPase activity of } \\
\text { chaperones of the HSP70/heat } \\
\text { shock protein } 70 \text { family. } \\
\text { Contributes to the ubiquitin- } \\
\text { dependent proteasomal } \\
\text { degradation of misfolded } \\
\text { proteins. }\end{array}$ & $\begin{array}{l}\text { (Gess et al., 2014; Lupo et } \\
\text { al., 2016) }\end{array}$ \\
\hline DNM2/CMT2M; CMTDIB* & $\begin{array}{l}\text { Plays an important role in } \\
\text { vesicular trafficking processes, in } \\
\text { particular endocytosis. Involved in } \\
\text { producing microtubule bundles. } \\
\text { Involved in cytokinesis. }\end{array}$ & $\begin{array}{l}\text { (Bitoun et al., 2008; } \\
\text { Echaniz-Laguna et al., 2007; } \\
\text { González-Jamett et al., } \\
\text { 2014; Haberlová et al., 2011; } \\
\text { Saghira et al., 2018) }\end{array}$ \\
\hline DYNC1H1/CMT2O* & $\begin{array}{l}\text { Acts as a motor for the } \\
\text { intracellular retrograde motility of } \\
\text { vesicles and organelles along } \\
\text { microtubules. Plays a role in } \\
\text { mitotic spindle assembly and } \\
\text { metaphase plate congression. }\end{array}$ & (Weedon et al., 2011) \\
\hline GARS1/CMT2D & $\begin{array}{l}\text { Catalyzes the attachment of } \\
\text { glycine to tRNA }\end{array}$ & $\begin{array}{l}\text { (Hamaguchi, Ishida, Iwasa, } \\
\text { Abe, \& Yamada, 2010; } \\
\text { James et al., 2006; Morelli et }\end{array}$ \\
\hline
\end{tabular}




\begin{tabular}{|l|l|l|}
\hline & & al., 2017; Motley et al., 2011; \\
W. Xie, Schimmel, \& Yang, \\
2006)
\end{tabular}




\begin{tabular}{|c|c|c|}
\hline & $\begin{array}{l}\text { Regulates numerous biological } \\
\text { processes including } \\
\text { phosphorylation and axonal } \\
\text { transport of neurofilament } \\
\text { proteins. }\end{array}$ & $\begin{array}{l}\text { et al., 2010; Tang et al., } \\
\text { 2005; Weeks et al., 2018) }\end{array}$ \\
\hline HSPB8/CMT2L* & $\begin{array}{l}\text { Displays temperature-dependent } \\
\text { chaperone activity. Forms } \\
\text { complex with BAG3. }\end{array}$ & $\begin{array}{l}\text { (Nicholson, Kennerson, } \\
\text { Brewer, Garbern, \& Shy, } \\
\text { 2009) }\end{array}$ \\
\hline IGHMBP2/CMT2S & $\begin{array}{l}5^{\prime} \text { to } 3^{\prime} \text { helicase that unwinds } \\
\text { RNA and DNA duplices. Acts as } \\
\text { a transcription regulator. }\end{array}$ & $\begin{array}{l}\text { (Cottenie et al., 2014; Dohrn } \\
\text { et al., 2017; Wagner et al., } \\
\text { 2015; Yuan et al., 2017) }\end{array}$ \\
\hline JPH1/CMT2K & $\begin{array}{l}\text { Contributes to the formation of } \\
\text { junctional membrane complexes } \\
\text { which link the plasma membrane } \\
\text { with the endoplasmic or } \\
\text { sarcoplasmic reticulum in } \\
\text { excitable cells. Provides a } \\
\text { structural foundation for } \\
\text { functional cross-talk between the } \\
\text { cell surface and intracellular } \\
\text { calcium release channels. }\end{array}$ & $\begin{array}{l}\text { (Kanwal \& Perveen, 2019; } \\
\text { Pla-Martín et al., 2015) }\end{array}$ \\
\hline $\mathrm{KIF} 1 \mathrm{~B} / \mathrm{CMT} 2 \mathrm{~A} 1^{*}$ & $\begin{array}{l}\text { Motor for anterograde transport of } \\
\text { mitochondria. }\end{array}$ & $\begin{array}{l}\text { (Bissar-Tadmouri et al., } \\
\text { 2004; Nakagawa \& } \\
\text { Takashima, 2003) }\end{array}$ \\
\hline KIF5A/CMT2* & $\begin{array}{l}\text { Microtubule-dependent motor } \\
\text { required for slow axonal transport } \\
\text { of neurofilament proteins. } \\
\text { Contributes to the vesicular } \\
\text { transport of VAPA, VAPB, } \\
\text { SURF4, RAB11A, RAB11B and } \\
\text { RTN3 proteins in neurons. }\end{array}$ & (Dohrn et al., 2017) \\
\hline LMNA/CMT2B1 & $\begin{array}{l}\text { Plays an important role in nuclear } \\
\text { assembly, chromatin } \\
\text { organization, nuclear membrane } \\
\text { and telomere dynamics. }\end{array}$ & $\begin{array}{l}\text { (Chaouch et al., 2003; De } \\
\text { Sandre-Giovannoli et al., } \\
\text { 2002; Dohrn et al., 2017; } \\
\text { Hamadouche et al., 2008; } \\
\text { Liang, Grogan, Ackerman, \& } \\
\text { Goodsell, 2016; Zhang et al., } \\
\text { 2010) }\end{array}$ \\
\hline
\end{tabular}




\begin{tabular}{|c|c|c|}
\hline LRSAM1/CMT2G & $\begin{array}{l}\text { E3 ubiquitin-protein ligase. } \\
\text { Bacterial recognition protein that } \\
\text { defends the cytoplasm from } \\
\text { invasive pathogens. Potential role } \\
\text { in mitophagy? }\end{array}$ & $\begin{array}{l}\text { (Dohrn et al., 2017; } \\
\text { Guernsey et al., 2010; } \\
\text { Nicolaou et al., 2013) }\end{array}$ \\
\hline MARS1/CMT2U & $\begin{array}{l}\text { Catalyzes the attachment of } \\
\text { methionine to tRNA. }\end{array}$ & $\begin{array}{l}\text { (Baets et al., 2014; Y. Sun } \\
\text { et al., 2017) }\end{array}$ \\
\hline MED25/CMT2B2 & $\begin{array}{l}\text { A coactivator involved in the } \\
\text { regulated transcription of nearly } \\
\text { all RNA polymerase II-dependent } \\
\text { genes. }\end{array}$ & $\begin{array}{l}\text { (Leal et al., 2018; Leal et al., } \\
\text { 2009; Tazir, Bellatache, } \\
\text { Nouioua, \& Vallat, 2013) }\end{array}$ \\
\hline MFN2/CMT2A2* & $\begin{array}{l}\text { Mitochondrial outer membrane } \\
\text { GTPase that mediates } \\
\text { mitochondrial clustering and } \\
\text { fusion. Involved in the clearance } \\
\text { of damaged mitochondria via } \\
\text { mitophagy. Potential roles in } \\
\text { mitochondria-ER contacts and } \\
\text { mitochondrial transport. }\end{array}$ & $\begin{array}{l}\text { (Ando et al., 2017; } \\
\text { Bannerman, Burns, Xu, } \\
\text { Miers, \& Pleasure, 2016; } \\
\text { Beręsewicz et al., 2017; } \\
\text { Bergamin et al., 2014; } \\
\text { Braathen, Sand, Lobato, } \\
\text { Hoyer, \& Russell, 2010; } \\
\text { Brožková et al., 2013; Calvo } \\
\text { et al., 2009; Cartoni \& } \\
\text { Martinou, 2009; Chapman, } \\
\text { Bennett, Ramesh, De Vos, \& } \\
\text { Grierson, 2013; B. O. Choi et } \\
\text { al., 2015; Del Bo et al., 2008; } \\
\text { Di Meglio et al., 2016; Dohrn } \\
\text { et al., 2017; El Fissi et al., } \\
\text { 2018; Engelfried et al., 2006; } \\
\text { Feely et al., 2011; Finsterer, } \\
\text { Fiorini, Scorza, \& Scorza, } \\
\text { 2018; lapadre et al., 2018; } \\
\text { Kijima et al., 2005; } \\
\text { Kotruchow, Kabzińska, } \\
\text { Hausmanowa-Petrusewicz, } \\
\text { \& Kochański, 2013; Larrea et } \\
\text { al., 2019; Lawson, Graham, } \\
\text { \& Flanigan, 2005; Loiseau et } \\
\text { al., 2007; Lv et al., 2013; Lv } \\
\text { et al., 2015; McCorquodale } \\
\text { et al., 2011; Milley et al., } \\
\text { 2018; Muglia et al., 2007; } \\
\text { Neupauerová, Grečmalová, } \\
\text { Seeman, \& Laššuthová, } \\
\text { 2016; Neusch et al., 2007; }\end{array}$ \\
\hline
\end{tabular}




\begin{tabular}{|c|c|c|}
\hline & & $\begin{array}{l}\text { Ouvrier \& Grew, 2010; S. Y. } \\
\text { Park et al., 2012; Rudnik- } \\
\text { Schöneborn et al., 2016; Tan } \\
\text { et al., 2016; Verhoeven, } \\
\text { Claeys, Züchner, et al., } \\
\text { 2006; Vielhaber et al., 2013; } \\
\text { Vital et al., 2012; Wang et } \\
\text { al., 2015; Werheid et al., } \\
\text { 2016; Y. Xie et al., 2016; Xu } \\
\text { et al., 2019; Zuchner et al., } \\
\text { 2006) }\end{array}$ \\
\hline MME/CMT2T & $\begin{array}{l}\text { Biologically important in the } \\
\text { destruction of opioid peptides. } \\
\text { Able to cleave angiotensins. } \\
\text { Involved in the degradation of } \\
\text { atrial natriuretic factor. }\end{array}$ & $\begin{array}{l}\text { (Auer-Grumbach et al., } \\
\text { 2016; Fujisawa et al., 2017) }\end{array}$ \\
\hline MORC2/CMT2Z & $\begin{array}{l}\text { Essential for epigenetic silencing } \\
\text { by the HUSH complex. }\end{array}$ & (Albulym et al., 2016) \\
\hline MPV17/CMT2EE* & $\begin{array}{l}\text { Non-selective channel that } \\
\text { modulates membrane potential } \\
\text { under normal conditions and } \\
\text { oxidative stress, and is involved } \\
\text { in mitochondrial homeostasis. } \\
\text { Involved in mitochondrial } \\
\text { deoxynucleoside triphosphates } \\
\text { pool homeostasis and } \\
\text { mitochondrial DNA maintenance. }\end{array}$ & (Y. R. Choi et al., 2015) \\
\hline $\begin{array}{l}\text { MPZ/CMT2J; CMTDID; } \\
\text { CMT1B }\end{array}$ & $\begin{array}{l}\text { Mediates adhesion between } \\
\text { adjacent myelin wraps and } \\
\text { ultimately drives myelin } \\
\text { compaction. }\end{array}$ & $\begin{array}{l}\text { (Avila et al., 2010; Bergamin } \\
\text { et al., 2014; Bienfait et al., } \\
\text { 2006; Bissar-Tadmouri et al., } \\
\text { 1999; Blanquet-Grossard et } \\
\text { al., 1996; Brozková, } \\
\text { Mazanec, Haberlová, } \\
\text { Sakmaryová, \& Seeman, } \\
\text { 2010; Chavada, Rao, } \\
\text { Martindale, \& Hadjivassiliou, } \\
\text { 2012; Choi et al., 2011; De } \\
\text { Jonghe et al., 1999; Dohrn et } \\
\text { al., 2017; Gallardo et al., } \\
\text { 2009; Hayasaka et al., 1993; } \\
\text { He et al., 2018; Høyer et al., } \\
\text { 2014; Høyer, Braathen, Eek, } \\
\text { Skjelbred, \& Russell, 2011; }\end{array}$ \\
\hline
\end{tabular}




\begin{tabular}{|c|c|c|}
\hline & & $\begin{array}{l}\text { Kleffner, Schirmacher, Gess, } \\
\text { Boentert, \& Young, 2010; } \\
\text { Kochański et al., 2004; } \\
\text { Kurihara et al., 2004; } \\
\text { Lagueny et al., 1999; Latour } \\
\text { et al., 1995; Laurà et al., } \\
\text { 2007; Leal et al., 2014; Lee } \\
\text { et al., 2008; Maeda et al., } \\
\text { 2012; Marttila et al., 2012; } \\
\text { Mazzeo et al., 2008; Milley et } \\
\text { al., 2018; Nelis et al., 1994; } \\
\text { Pham-Dinh et al., 1993; } \\
\text { Quattrini et al., 1999; Roa et } \\
\text { al., 1996; Rosberg, Alvarez, } \\
\text { Krarup, \& Moldovan, 2013; } \\
\text { Rudnik-Schöneborn et al., } \\
\text { 2016; Sabet et al., 2006; } \\
\text { Sanmaneechai et al., 2015; } \\
\text { Senderek et al., 2000; } \\
\text { Silander et al., 1996; } \\
\text { Speevak \& Farrell, 2013; } \\
\text { Tokuda et al., 2015; Wang et } \\
\text { al., 2015; Werheid et al., } \\
\text { 2016; Xu et al., 2019) }\end{array}$ \\
\hline MT-ATP6/CMT2* & $\begin{array}{l}\text { Mitochondrial membrane ATP } \\
\text { synthase. Key component of the } \\
\text { proton channel. }\end{array}$ & (Pitceathly et al., 2012) \\
\hline NAGLU/CMT2V & $\begin{array}{l}\text { Involved in the degradation of } \\
\text { heparan sulfate. }\end{array}$ & (Tétreault et al., 2015) \\
\hline $\mathrm{NEFH} / \mathrm{CMT} 2 \mathrm{CC}^{*}$ & $\begin{array}{l}\text { Component of neurofilaments, } \\
\text { the most abundant cytoskeletal } \\
\text { component of myelinated axons. }\end{array}$ & (Bian et al., 2018) \\
\hline NEFL/CMT2E* & $\begin{array}{l}\text { Component of neurofilaments, } \\
\text { the most abundant cytoskeletal } \\
\text { component of myelinated axons. } \\
\text { Regulates mitochondrial } \\
\text { morphology. }\end{array}$ & $\begin{array}{l}\text { (Berciano et al., 2016; } \\
\text { Bhagavati, Maccabee, \& Xu, } \\
\text { 2009; Dohrn et al., 2017; } \\
\text { Fabrizi et al., 2007; Fu \& } \\
\text { Yuan, 2018; Horga et al., } \\
\text { 2017; Luo et al., 2003; } \\
\text { Lupski, 2000; Miltenberger- } \\
\text { Miltenyi et al., 2007; Shin et } \\
\text { al., 2008; Werheid et al., } \\
\text { 2016; Xu et al., 2019) }\end{array}$ \\
\hline
\end{tabular}




\begin{tabular}{|c|c|c|}
\hline PNKP/CMT2B2 & $\begin{array}{l}\text { Plays a key role in the repair of } \\
\text { DNA damage, functioning as part } \\
\text { of both the non-homologous end- } \\
\text { joining and base excision repair } \\
\text { pathways. }\end{array}$ & (Leal et al., 2018) \\
\hline RAB7A/CMT2B* & $\begin{array}{l}\text { Key regulator in endo-lysosomal } \\
\text { trafficking. Plays roles in growth- } \\
\text { factor-mediated cell signaling, } \\
\text { nutrient-transportor mediated } \\
\text { nutrient uptake, neurotrophin } \\
\text { transport in the axons of neurons } \\
\text { and lipid metabolism. Regulates } \\
\text { mitochondrial fission, mitophagy, } \\
\text { and mitochondria-lysosome } \\
\text { tethering. }\end{array}$ & $\begin{array}{l}\text { (Manganelli et al., 2015; } \\
\text { Meggouh, Bienfait, } \\
\text { Weterman, de Visser, \& } \\
\text { Baas, 2006; Zhang et al., } \\
\text { 2010) }\end{array}$ \\
\hline SPG11/CMT2X* & $\begin{array}{l}\text { Plays a role in neurite plasticity } \\
\text { by maintaining cytoskeleton } \\
\text { stability and regulating synaptic } \\
\text { vesicle transport. }\end{array}$ & (Montecchiani et al., 2016) \\
\hline TRIM2/CMT2R* & $\begin{array}{l}\text { E3 ubiquitin-protein ligase that } \\
\text { mediates the ubiquitination of } \\
\text { NEFL and of phosphorylated } \\
\text { BCL2L11. }\end{array}$ & $\begin{array}{l}\text { (Baets et al., 2014; Pehlivan } \\
\text { et al., 2015) }\end{array}$ \\
\hline TRPV4/CMT2C* & $\begin{array}{l}\text { Non-selective calcium permeant } \\
\text { cation channel involved in } \\
\text { osmotic sensitivity and } \\
\text { mechanosensitivity. Some data } \\
\text { supporting a role in regulating } \\
\text { mitochondrial motility. }\end{array}$ & $\begin{array}{l}\text { (Deng et al., 2010; Dohrn et } \\
\text { al., 2017; Klein et al., 2003; } \\
\text { Manganelli et al., 2015) }\end{array}$ \\
\hline VCP/CMT2Y* & $\begin{array}{l}\text { Necessary for the fragmentation } \\
\text { of Golgi stacks during mitosis and } \\
\text { for their reassembly after mitosis. } \\
\text { Involved in the formation of the } \\
\text { transitional endoplasmic } \\
\text { reticulum. Plays a role in the } \\
\text { regulation of stress granule } \\
\text { clearance. Involved in DNA } \\
\text { damage response. Essential for } \\
\text { the maturation of ubiquitin- } \\
\text { containing autophagosomes and }\end{array}$ & (Gonzalez et al., 2014) \\
\hline
\end{tabular}




\begin{tabular}{|c|c|c|}
\hline & $\begin{array}{l}\text { the clearance of ubiquitinated } \\
\text { protein by autophagy and } \\
\text { mitophagy. }\end{array}$ & \\
\hline C1ORF194/CMTDI & $\begin{array}{l}\text { May affect intracellular } \mathrm{Ca}^{2+} \\
\text { homeostasis. }\end{array}$ & (S. C. Sun et al., 2019) \\
\hline GNB4/CMTDIF & $\begin{array}{l}\text { Modulator/transducer in various } \\
\text { transmembrane signaling } \\
\text { systems. }\end{array}$ & $\begin{array}{l}\text { (Baets et al., 2014; Miura et } \\
\text { al., 2017; Soong et al., 2013) }\end{array}$ \\
\hline INF2/CMTDIE* & $\begin{array}{l}\text { Mediates actin polymerization at } \\
\text { ER-mitochondria contact sites. } \\
\text { Regulates mitochondrial } \\
\text { morphology and motility. }\end{array}$ & $\begin{array}{l}\text { (Boyer, Benoit, et al., 2011; } \\
\text { Boyer, Nevo, et al., 2011; } \\
\text { Caridi et al., 2014; Dohrn et } \\
\text { al., 2017; Echaniz-Laguna \& } \\
\text { Latour, 2019; Fu et al., } 2019 \\
\text { Jin et al., 2015; Mademan et } \\
\text { al., 2013; H. J. Park et al., } \\
\text { 2014; Rodriguez et al., 2013 } \\
\text { Vallat, Mathis, \& Funalot, } \\
\text { 2013; Werheid et al., 2016) }\end{array}$ \\
\hline SLC12A6/CMTDI & $\begin{array}{l}\text { Mediates electroneutral } \\
\text { potassium-chloride cotransport. }\end{array}$ & (Lupo et al., 2016) \\
\hline YARS1/CMTDIC & $\begin{array}{l}\text { Catalyzes the attachment of } \\
\text { tyrosine to its corresponding } \\
\text { tRNA }\end{array}$ & $\begin{array}{l}\text { (Jordanova et al., 2006; W. } \\
\text { Xie, Nangle, Zhang, } \\
\text { Schimmel, \& Yang, 2007) }\end{array}$ \\
\hline 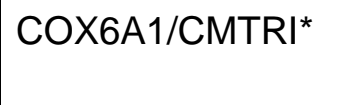 & $\begin{array}{l}\text { A subunit of the cytochrome } c \\
\text { oxidase complex }\end{array}$ & (Tamiya et al., 2014) \\
\hline KARS1/CMTRIB & $\begin{array}{l}\text { Catalyzes the aminoacylation of } \\
\text { tRNA-Lys in the cytoplasm and } \\
\text { mitochondria }\end{array}$ & (McLaughlin et al., 2010) \\
\hline PLEKHG5/CMTRIC & $\begin{array}{l}\text { Activates the nuclear factor } \\
\text { kappa B (NFKB1) signaling } \\
\text { pathway. Also implicated in distal } \\
\text { spinal muscular atrophy. }\end{array}$ & (H. J. Kim et al., 2013) \\
\hline
\end{tabular}




\section{Literature Cited}

Albulym, O. M., Kennerson, M. L., Harms, M. B., Drew, A. P., Siddell, A. H., Auer-Grumbach, M., .. Nicholson, G. A. (2016). MORC2 mutations cause axonal Charcot-Marie-Tooth disease with pyramidal signs. Ann Neurol, 79(3), 419-427. doi:10.1002/ana.24575

Ammar, N., Nelis, E., Merlini, L., Barisić, N., Amouri, R., Ceuterick, C., . . De Jonghe, P. (2003). Identification of novel GDAP1 mutations causing autosomal recessive Charcot-Marie-Tooth disease. Neuromuscul Disord, 13(9), 720-728. doi:10.1016/s0960-8966(03)00093-2

Amornvit, J., Yalvac, M. E., Chen, L., \& Sahenk, Z. (2017). A novel p.T139M mutation in HSPB1 highlighting the phenotypic spectrum in a family. Brain Behav, 7(8), e00774. doi:10.1002/brb3.774

Ando, M., Hashiguchi, A., Okamoto, Y., Yoshimura, A., Hiramatsu, Y., Yuan, J., . . Takashima, H. (2017). Clinical and genetic diversities of Charcot-Marie-Tooth disease with MFN2 mutations in a large case study. J Peripher Nerv Syst, 22(3), 191-199. doi:10.1111/jns.12228

Auer-Grumbach, M., Fischer, C., Papić, L., John, E., Plecko, B., Bittner, R. E., . . Janecke, A. R. (2008). Two novel mutations in the GDAP1 and PRX genes in early onset Charcot-Marie-Tooth syndrome. Neuropediatrics, 39(1), 33-38. doi:10.1055/s-2008-1077085

Auer-Grumbach, M., Toegel, S., Schabhüttl, M., Weinmann, D., Chiari, C., Bennett, D. L. H., . . Senderek, J. (2016). Rare Variants in MME, Encoding Metalloprotease Neprilysin, Are Linked to Late-Onset Autosomal-Dominant Axonal Polyneuropathies. Am J Hum Genet, 99(3), 607-623. doi:10.1016/j.ajhg.2016.07.008

Auranen, M., Ylikallio, E., Toppila, J., Somer, M., Kiuru-Enari, S., \& Tyynismaa, H. (2013). Dominant GDAP1 founder mutation is a common cause of axonal Charcot-Marie-Tooth disease in Finland. Neurogenetics, 14(2), 123-132. doi:10.1007/s10048-013-0358-9

Avila, R. L., D'Antonio, M., Bachi, A., Inouye, H., Feltri, M. L., Wrabetz, L., \& Kirschner, D. A. (2010). P0 (protein zero) mutation S34C underlies instability of internodal myelin in S63C mice. J Biol Chem, 285(53), 42001-42012. doi:10.1074/jbc.M110.166967

Baets, J., De Jonghe, P., \& Timmerman, V. (2014). Recent advances in Charcot-Marie-Tooth disease. Curr Opin Neurol, 27(5), 532-540. doi:10.1097/wco.0000000000000131

Baloh, R. H., Schmidt, R. E., Pestronk, A., \& Milbrandt, J. (2007). Altered axonal mitochondrial transport in the pathogenesis of Charcot-Marie-Tooth disease from mitofusin 2 mutations. J Neurosci, 27(2), 422-430. doi:10.1523/JNEUROSCI.4798-06.2007

Bannerman, P., Burns, T., Xu, J., Miers, L., \& Pleasure, D. (2016). Mice Hemizygous for a Pathogenic Mitofusin-2 Allele Exhibit Hind Limb/Foot Gait Deficits and Phenotypic Perturbations in Nerve and Muscle. PLoS One, 11(12), e0167573. doi:10.1371/journal.pone.0167573

Bansagi, B., Antoniadi, T., Burton-Jones, S., Murphy, S. M., McHugh, J., Alexander, M., ... Horvath, R. (2015). Genotype/phenotype correlations in AARS-related neuropathy in a cohort of patients from the United Kingdom and Ireland. J Neurol, 262(8), 1899-1908. doi:10.1007/s00415-0157778-4

Baránková, L., Vyhnálková, E., Züchner, S., Mazanec, R., Sakmaryová, I., Vondrácek, P., . . Seeman, P. (2007). GDAP1 mutations in Czech families with early-onset CMT. Neuromuscul Disord, 17(6), 482-489. doi:10.1016/j.nmd.2007.02.010

Baxter, R. V., Ben Othmane, K., Rochelle, J. M., Stajich, J. E., Hulette, C., Dew-Knight, S., . . Vance, J. M. (2002). Ganglioside-induced differentiation-associated protein-1 is mutant in Charcot-MarieTooth disease type 4A/8q21. Nat Genet, 30(1), 21-22. doi:10.1038/ng796

Bayraktar, S., Nehrig, J., Menis, E., Karli, K., Janning, A., Struk, T., . . Wedlich-Söldner, R. (2020). A Deregulated Stress Response Underlies Distinct INF2-Associated Disease Profiles. J Am Soc Nephrol, 31(6), 1296-1313. doi:10.1681/asn.2019111174 
Berciano, J., Peeters, K., García, A., López-Alburquerque, T., Gallardo, E., Hernández-Fabián, A., . . Jordanova, A. (2016). NEFL N98S mutation: another cause of dominant intermediate CharcotMarie-Tooth disease with heterogeneous early-onset phenotype. J Neurol, 263(2), 361-369. doi:10.1007/s00415-015-7985-z

Beręsewicz, M., Boratyńska-Jasińska, A., Charzewski, Ł., Kawalec, M., Kabzińska, D., Kochański, A., . . . Zabłocka, B. (2017). The Effect of a Novel c.820C >T (Arg274Trp) Mutation in the Mitofusin 2 Gene on Fibroblast Metabolism and Clinical Manifestation in a Patient. PLoS One, 12(1), e0169999. doi:10.1371/journal.pone.0169999

Bergamin, G., Boaretto, F., Briani, C., Pegoraro, E., Cacciavillani, M., Martinuzzi, A., ... Mostacciuolo, M. L. (2014). Mutation analysis of MFN2, GJB1, MPZ and PMP22 in Italian patients with axonal Charcot-Marie-Tooth disease. Neuromolecular Med, 16(3), 540-550. doi:10.1007/s12017-0148307-9

Bernard-Marissal, N., van Hameren, G., Juneja, M., Pellegrino, C., Louhivuori, L., Bartesaghi, L., . . Chrast, R. (2019). Altered interplay between endoplasmic reticulum and mitochondria in Charcot-Marie-Tooth type 2A neuropathy. Proc Natl Acad Sci U S A, 116(6), 2328-2337. doi:10.1073/pnas.1810932116

Bhagavati, S., Maccabee, P. J., \& Xu, W. (2009). The neurofilament light chain gene (NEFL) mutation Pro22Ser can be associated with mixed axonal and demyelinating neuropathy. J Clin Neurosci, 16(6), 830-831. doi:10.1016/j.jocn.2008.08.030

Bian, X., Lin, P., Li, J., Long, F., Duan, R., Yuan, Q., . . Liu, Q. (2018). Whole-Genome Linkage Analysis with Whole-Exome Sequencing Identifies a Novel Frameshift Variant in NEFH in a Chinese Family with Charcot-Marie-Tooth 2: A Novel Variant in NEFH for Charcot-Marie-Tooth 2. Neurodegener Dis, 18(2-3), 74-83. doi:10.1159/000487754

Bienfait, H. M., Faber, C. G., Baas, F., Gabreëls-Festen, A. A., Koelman, J. H., Hoogendijk, J. E., . . . de Visser, M. (2006). Late onset axonal Charcot-Marie-Tooth phenotype caused by a novel myelin protein zero mutation. J Neurol Neurosurg Psychiatry, 77(4), 534-537. doi:10.1136/jnnp.2005.073437

Bissar-Tadmouri, N., Gulsen-Parman, Y., Latour, P., Deymeer, F., Serdaroglu, P., Vandenberghe, A., \& Battaloglu, E. (1999). Two novel mutations in the MPZ gene coding region in Charcot-MarieTooth type 1 patients of Turkish origin: S54P, [I30del; GVYI29ins]. Hum Mutat, 14(5), 449. doi:10.1002/(sici)1098-1004(199911)14:5<449::Aid-humu17>3.0.Co;2-h

Bissar-Tadmouri, N., Nelis, E., Züchner, S., Parman, Y., Deymeer, F., Serdaroglu, P., . . Battaloglu, E. (2004). Absence of KIF1B mutation in a large Turkish CMT2A family suggests involvement of a second gene. Neurology, 62(9), 1522-1525. doi:10.1212/01.wnl.0000123253.57555.3a

Bitoun, M., Stojkovic, T., Prudhon, B., Maurage, C. A., Latour, P., Vermersch, P., \& Guicheney, P. (2008). A novel mutation in the dynamin 2 gene in a Charcot-Marie-Tooth type 2 patient: clinical and pathological findings. Neuromuscul Disord, 18(4), 334-338. doi:10.1016/j.nmd.2008.01.005

Blanquet-Grossard, F., Pham-Dinh, D., Dautigny, A., Latour, P., Bonnebouche, C., Diraison, P., . . . Vandenberghe, A. (1996). Charcot-Marie-Tooth type 1B neuropathy: a mutation at the single glycosylation site in the major peripheral myelin glycoprotein Po. Hum Mutat, 8(2), 185-186. doi:10.1002/(sici)1098-1004(1996)8:2<185::Aid-humu13>3.0.Co;2-z

Boyer, O., Benoit, G., Gribouval, O., Nevo, F., Tete, M. J., Dantal, J., . . Antignac, C. (2011). Mutations in INF2 are a major cause of autosomal dominant focal segmental glomerulosclerosis. J Am Soc Nephrol, 22(2), 239-245. doi:10.1681/ASN.2010050518

Boyer, O., Nevo, F., Plaisier, E., Funalot, B., Gribouval, O., Benoit, G., . . Mollet, G. (2011). INF2 mutations in Charcot-Marie-Tooth disease with glomerulopathy. N Engl J Med, 365(25), $2377-$ 2388. doi:10.1056/NEJMoa1109122 
Braathen, G. J., Høyer, H., Busk $\varnothing$, L., Tveten, K., Skjelbred, C. F., \& Russell, M. B. (2016). Variants in the genes DCTN2, DNAH10, LRIG3, and MYO1A are associated with intermediate Charcot-MarieTooth disease in a Norwegian family. Acta Neurol Scand, 134(1), 67-75. doi:10.1111/ane.12515

Braathen, G. J., Sand, J. C., Lobato, A., Hoyer, H., \& Russell, M. B. (2010). MFN2 point mutations occur in $3.4 \%$ of Charcot-Marie-Tooth families. An investigation of 232 Norwegian CMT families. BMC Med Genet, 11, 48. doi:10.1186/1471-2350-11-48

Brozková, D., Mazanec, R., Haberlová, J., Sakmaryová, I., \& Seeman, P. (2010). Clinical and in silico evidence for and against pathogenicity of 11 new mutations in the MPZ gene. Clin Genet, 78(1), 81-87. doi:10.1111/j.1399-0004.2010.01423.x

Brožková, D., Posádka, J., Laššuthová, P., Mazanec, R., Haberlová, J., Sišková, D., . . Seeman, P. (2013). Spectrum and frequencies of mutations in the MFN2 gene and its phenotypical expression in Czech hereditary motor and sensory neuropathy type II patients. Mol Med Rep, 8(6), 1779-1784. doi:10.3892/mmr.2013.1730

Calvo, J., Funalot, B., Ouvrier, R. A., Lazaro, L., Toutain, A., De Mas, P., . . Magdelaine, C. (2009). Genotype-phenotype correlations in Charcot-Marie-Tooth disease type 2 caused by mitofusin 2 mutations. Arch Neurol, 66(12), 1511-1516. doi:10.1001/archneurol.2009.284

Cardanho-Ramos, C., Faria-Pereira, A., \& Morais, V. A. (2020). Orchestrating mitochondria in neurons: Cytoskeleton as the conductor. Cytoskeleton (Hoboken), 77(3-4), 65-75. doi:10.1002/cm.21585

Caridi, G., Lugani, F., Dagnino, M., Gigante, M., Iolascon, A., Falco, M., . . . Ghiggeri, G. M. (2014). Novel INF2 mutations in an Italian cohort of patients with focal segmental glomerulosclerosis, renal failure and Charcot-Marie-Tooth neuropathy. Nephrol Dial Transplant, 29 Suppl 4, iv80-86. doi:10.1093/ndt/gfu071

Cartoni, R., \& Martinou, J. C. (2009). Role of mitofusin 2 mutations in the physiopathology of CharcotMarie-Tooth disease type 2A. Exp Neurol, 218(2), 268-273. doi:10.1016/j.expneurol.2009.05.003

Cassereau, J., Chevrollier, A., Bonneau, D., Verny, C., Procaccio, V., Reynier, P., \& Ferré, M. (2011). A locus-specific database for mutations in GDAP1 allows analysis of genotype-phenotype correlations in Charcot-Marie-Tooth diseases type 4A and 2K. Orphanet J Rare Dis, 6, 87. doi:10.1186/1750-1172-6-87

Chada, S. R., \& Hollenbeck, P. J. (2004). Nerve growth factor signaling regulates motility and docking of axonal mitochondria. Curr Biol, 14(14), 1272-1276. doi:10.1016/j.cub.2004.07.027

Chakrabarti, R., Ji, W. K., Stan, R. V., de Juan Sanz, J., Ryan, T. A., \& Higgs, H. N. (2018). INF2-mediated actin polymerization at the ER stimulates mitochondrial calcium uptake, inner membrane constriction, and division. J Cell Biol, 217(1), 251-268. doi:10.1083/jcb.201709111

Chaouch, M., Allal, Y., De Sandre-Giovannoli, A., Vallat, J. M., Amer-el-Khedoud, A., Kassouri, N., ... Grid, D. (2003). The phenotypic manifestations of autosomal recessive axonal Charcot-Marie-Tooth due to a mutation in Lamin A/C gene. Neuromuscul Disord, 13(1), 60-67. doi:10.1016/s09608966(02)00196-7

Chapman, A. L., Bennett, E. J., Ramesh, T. M., De Vos, K. J., \& Grierson, A. J. (2013). Axonal Transport Defects in a Mitofusin 2 Loss of Function Model of Charcot-Marie-Tooth Disease in Zebrafish. PLoS One, 8(6), e67276. doi:10.1371/journal.pone.0067276

Chaudhry, R., Kidambi, A., Brewer, M. H., Antonellis, A., Mathews, K., Nicholson, G., \& Kennerson, M. (2013). Re-analysis of an original CMTX3 family using exome sequencing identifies a known BSCL2 mutation. Muscle Nerve, 47(6), 922-924. doi:10.1002/mus.23743

Chavada, G., Rao, D. G., Martindale, J., \& Hadjivassiliou, M. (2012). A novel MPZ gene mutation in exon 2 causing late-onset demyelinating Charcot-Marie-Tooth disease. J Clin Neuromuscul Dis, 13(4), 206-208. doi:10.1097/CND.0b013e3182461a83 
Choi, B. O., Kim, S. B., Kanwal, S., Hyun, Y. S., Park, S. W., Koo, H., . . Chung, K. W. (2011). MPZ mutation in an early-onset Charcot-Marie-Tooth disease type 1B family by genome-wide linkage analysis. Int J Mol Med, 28(3), 389-396. doi:10.3892/ijmm.2011.678

Choi, B. O., Nakhro, K., Park, H. J., Hyun, Y. S., Lee, J. H., Kanwal, S., . . Chung, K. W. (2015). A cohort study of MFN2 mutations and phenotypic spectrums in Charcot-Marie-Tooth disease 2A patients. Clin Genet, 87(6), 594-598. doi:10.1111/cge.12432

Choi, Y. R., Hong, Y. B., Jung, S. C., Lee, J. H., Kim, Y. J., Park, H. J., . . Choi, B. O. (2015). A novel homozygous MPV17 mutation in two families with axonal sensorimotor polyneuropathy. $B M C$ Neurol, 15, 179. doi:10.1186/s12883-015-0430-1

Chung, K. W., Kim, S. B., Cho, S. Y., Hwang, S. J., Park, S. W., Kang, S. H., . . Choi, B. O. (2008). Distal hereditary motor neuropathy in Korean patients with a small heat shock protein 27 mutation. Exp Mol Med, 4O(3), 304-312. doi:10.3858/emm.2008.40.3.304

Chung, K. W., Kim, S. B., Park, K. D., Choi, K. G., Lee, J. H., Eun, H. W., . . Choi, B. O. (2006). Early onset severe and late-onset mild Charcot-Marie-Tooth disease with mitofusin 2 (MFN2) mutations. Brain, 129(Pt 8), 2103-2118. doi:10.1093/brain/awl174

Cioni, J. M., Lin, J. Q., Holtermann, A. V., Koppers, M., Jakobs, M. A. H., Azizi, A., .. Holt, C. E. (2019). Late Endosomes Act as mRNA Translation Platforms and Sustain Mitochondria in Axons. Cell, 176(1-2), 56-72 e15. doi:10.1016/j.cell.2018.11.030

Cottenie, E., Kochanski, A., Jordanova, A., Bansagi, B., Zimon, M., Horga, A., . . Houlden, H. (2014). Truncating and missense mutations in IGHMBP2 cause Charcot-Marie Tooth disease type 2. Am J Hum Genet, 95(5), 590-601. doi:10.1016/j.ajhg.2014.10.002

Cuesta, A., Pedrola, L., Sevilla, T., García-Planells, J., Chumillas, M. J., Mayordomo, F., . . Palau, F. (2002). The gene encoding ganglioside-induced differentiation-associated protein 1 is mutated in axonal Charcot-Marie-Tooth type 4A disease. Nat Genet, 30(1), 22-25. doi:10.1038/ng798

De Jonghe, P., Timmerman, V., Ceuterick, C., Nelis, E., De Vriendt, E., Löfgren, A., . . Van Broeckhoven, C. (1999). The Thr124Met mutation in the peripheral myelin protein zero (MPZ) gene is associated with a clinically distinct Charcot-Marie-Tooth phenotype. Brain, 122 ( Pt 2), 281-290. doi:10.1093/brain/122.2.281

De Sandre-Giovannoli, A., Chaouch, M., Kozlov, S., Vallat, J. M., Tazir, M., Kassouri, N., . . Lévy, N. (2002). Homozygous defects in LMNA, encoding lamin A/C nuclear-envelope proteins, cause autosomal recessive axonal neuropathy in human (Charcot-Marie-Tooth disorder type 2 ) and mouse. Am J Hum Genet, 70(3), 726-736. doi:10.1086/339274

Del Bo, R., Moggio, M., Rango, M., Bonato, S., D'Angelo, M. G., Ghezzi, S., . . Comi, G. P. (2008). Mutated mitofusin 2 presents with intrafamilial variability and brain mitochondrial dysfunction. Neurology, 71(24), 1959-1966. doi:10.1212/01.wnl.0000327095.32005.a4

Deng, H. X., Klein, C. J., Yan, J., Shi, Y., Wu, Y., Fecto, F., . . Siddique, T. (2010). Scapuloperoneal spinal muscular atrophy and CMT2C are allelic disorders caused by alterations in TRPV4. Nat Genet, 42(2), 165-169. doi:10.1038/ng.509

Detmer, S. A., \& Chan, D. C. (2007). Complementation between mouse Mfn1 and Mfn2 protects mitochondrial fusion defects caused by CMT2A disease mutations. J Cell Biol, 176(4), 405-414. doi:10.1083/jcb.200611080

Devine, M. J., \& Kittler, J. T. (2018). Mitochondria at the neuronal presynapse in health and disease. Nat Rev Neurosci, 19(2), 63-80. doi:10.1038/nrn.2017.170

Di Maria, E., Gulli, R., Balestra, P., Cassandrini, D., Pigullo, S., Doria-Lamba, L., . . Bellone, E. (2004). A novel mutation of GDAP1 associated with Charcot-Marie-Tooth disease in three Italian families: evidence for a founder effect. J Neurol Neurosurg Psychiatry, 75(10), 1495-1498. doi:10.1136/jnnp.2003.028100 
Di Meglio, C., Bonello-Palot, N., Boulay, C., Milh, M., Ovaert, C., Levy, N., \& Chabrol, B. (2016). Clinical and allelic heterogeneity in a pediatric cohort of 11 patients carrying MFN2 mutation. Brain Dev, 38(5), 498-506. doi:10.1016/j.braindev.2015.11.006

Dohrn, M. F., Glöckle, N., Mulahasanovic, L., Heller, C., Mohr, J., Bauer, C., . . . Biskup, S. (2017). Frequent genes in rare diseases: panel-based next generation sequencing to disclose causal mutations in hereditary neuropathies. J Neurochem, 143(5), 507-522. doi:10.1111/jnc.14217

Echaniz-Laguna, A., \& Latour, P. (2019). A cryptic splicing mutation in the INF2 gene causing CharcotMarie-Tooth disease with minimal glomerular dysfunction. J Peripher Nerv Syst, 24(1), 120-124. doi:10.1111/jns.12308

Echaniz-Laguna, A., Nicot, A. S., Carré, S., Franques, J., Tranchant, C., Dondaine, N., . . Laporte, J. (2007). Subtle central and peripheral nervous system abnormalities in a family with centronuclear myopathy and a novel dynamin 2 gene mutation. Neuromuscul Disord, 17(11-12), 955-959. doi:10.1016/j.nmd.2007.06.467

El Fissi, N., Rojo, M., Aouane, A., Karatas, E., Poliacikova, G., David, C., . . Rival, T. (2018). Mitofusin gain and loss of function drive pathogenesis in Drosophila models of CMT2A neuropathy. EMBO Rep, 19(8). doi:10.15252/embr.201745241

Engelfried, K., Vorgerd, M., Hagedorn, M., Haas, G., Gilles, J., Epplen, J. T., \& Meins, M. (2006). CharcotMarie-Tooth neuropathy type 2A: novel mutations in the mitofusin 2 gene (MFN2). BMC Med Genet, 7, 53. doi:10.1186/1471-2350-7-53

Fabrizi, G. M., Cavallaro, T., Angiari, C., Cabrini, I., Taioli, F., Malerba, G., . . Rizzuto, N. (2007). CharcotMarie-Tooth disease type 2E, a disorder of the cytoskeleton. Brain, 130(Pt 2), 394-403. doi:10.1093/brain/awl284

Feely, S. M., Laura, M., Siskind, C. E., Sottile, S., Davis, M., Gibbons, V. S., . . Shy, M. E. (2011). MFN2 mutations cause severe phenotypes in most patients with CMT2A. Neurology, 76(20), 16901696. doi:10.1212/WNL.0b013e31821a441e

Finsterer, J., Fiorini, A. C., Scorza, C. A., \& Scorza, F. A. (2018). CMT2 due to homozygous MFN2 variants is a multiorgan mitochondrial disorder. Eur J Paediatr Neurol, 22(5), 889-891. doi:10.1016/j.ejpn.2018.04.012

Fu, J., Ma, M., Pang, M., Yang, L., Li, G., Song, J., \& Zhang, J. (2019). [Analysis of a pedigree with autosomal dominant intermediate Charcot-Marie-Tooth disease type $E$ and nephropathy]. Zhonghua Yi Xue Yi Chuan Xue Za Zhi, 36(9), 918-921. doi:10.3760/cma.j.issn.10039406.2019.09.016

Fu, J., \& Yuan, Y. (2018). A novel homozygous nonsense mutation in NEFL causes autosomal recessive Charcot-Marie-Tooth disease. Neuromuscul Disord, 28(1), 44-47. doi:10.1016/j.nmd.2017.09.018

Fujisawa, M., Sano, Y., Omoto, M., Ogasawara, J. I., Koga, M., Takashima, H., \& Kanda, T. (2017). [Charcot-Marie-Tooth disease type 2 caused by homozygous MME gene mutation superimposed by chronic inflammatory demyelinating polyneuropathy]. Rinsho Shinkeigaku, 57(9), 515-520. doi:10.5692/clinicalneurol.cn-001036

Fusco, C., Ucchino, V., Barbon, G., Bonini, E., Mostacciuolo, M. L., Frattini, D., .. . Giustina, E. D. (2011). The homozygous ganglioside-induced differentiation-associated protein 1 mutation c.373C > T causes a very early-onset neuropathy: case report and literature review. J Child Neurol, 26(1), 49-57. doi:10.1177/0883073810373142

Gallardo, E., García, A., Ramón, C., Maraví, E., Infante, J., Gastón, I., . . . Berciano, J. (2009). CharcotMarie-Tooth disease type 2J with MPZ Thr124Met mutation: clinico-electrophysiological and MRI study of a family. J Neurol, 256(12), 2061-2071. doi:10.1007/s00415-009-5251-y

Gess, B., Auer-Grumbach, M., Schirmacher, A., Strom, T., Zitzelsberger, M., Rudnik-Schöneborn, S., . . . Senderek, J. (2014). HSJ1-related hereditary neuropathies: novel mutations and extended clinical spectrum. Neurology, 83(19), 1726-1732. doi:10.1212/wnl.0000000000000966 
González-Jamett, A. M., Haro-Acuña, V., Momboisse, F., Caviedes, P., Bevilacqua, J. A., \& Cárdenas, A. M. (2014). Dynamin-2 in nervous system disorders. J Neurochem, 128(2), 210-223. doi:10.1111/jnc.12455

Gonzalez, M. A., Feely, S. M., Speziani, F., Strickland, A. V., Danzi, M., Bacon, C., . . Shy, M. E. (2014). A novel mutation in VCP causes Charcot-Marie-Tooth Type 2 disease. Brain, 137(Pt 11), 28972902. doi:10.1093/brain/awu224

Guernsey, D. L., Jiang, H., Bedard, K., Evans, S. C., Ferguson, M., Matsuoka, M., . . Samuels, M. E. (2010). Mutation in the gene encoding ubiquitin ligase LRSAM1 in patients with Charcot-Marie-Tooth disease. PLoS Genet, 6(8). doi:10.1371/journal.pgen.1001081

Haberlová, J., Mazanec, R., Ridzoň, P., Baránková, L., Nürnberg, G., Nürnberg, P., . . Rautenstrauss, B. (2011). Phenotypic variability in a large Czech family with a dynamin 2-associated CharcotMarie-Tooth neuropathy. J Neurogenet, 25(4), 182-188. doi:10.3109/01677063.2011.627484

Hamadouche, T., Poitelon, Y., Genin, E., Chaouch, M., Tazir, M., Kassouri, N., . . Delague, V. (2008). Founder effect and estimation of the age of the c.892C >T (p.Arg298Cys) mutation in LMNA associated to Charcot-Marie-Tooth subtype CMT2B1 in families from North Western Africa. Ann Hum Genet, 72(Pt 5), 590-597. doi:10.1111/j.1469-1809.2008.00456.x

Hamaguchi, A., Ishida, C., Iwasa, K., Abe, A., \& Yamada, M. (2010). Charcot-Marie-Tooth disease type 2D with a novel glycyl-tRNA synthetase gene (GARS) mutation. J Neurol, 257(7), 1202-1204. doi:10.1007/s00415-010-5491-x

Hayasaka, K., Himoro, M., Sato, W., Takada, G., Uyemura, K., Shimizu, N., . . Chance, P. F. (1993). Charcot-Marie-Tooth neuropathy type $1 \mathrm{~B}$ is associated with mutations of the myelin P0 gene. Nat Genet, 5(1), 31-34. doi:10.1038/ng0993-31

He, J., Guo, L., Xu, G., Xu, L., Lin, S., Chen, W., \& Wang, N. (2018). Clinical and genetic investigation in Chinese patients with demyelinating Charcot-Marie-Tooth disease. J Peripher Nerv Syst, 23(4), 216-226. doi:10.1111/jns.12277

Ho, C. C., Tai, S. M., Lee, E. C., Mak, T. S., Liu, T. K., Tang, V. W., \& Poon, W. T. (2017). Rapid Identification of Pathogenic Variants in Two Cases of Charcot-Marie-Tooth Disease by Gene-Panel Sequencing. Int J Mol Sci, 18(4). doi:10.3390/ijms18040770

Hong, Y. B., Kang, J., Kim, J. H., Lee, J., Kwak, G., Hyun, Y. S., . . Chung, K. W. (2016). DGAT2 Mutation in a Family with Autosomal-Dominant Early-Onset Axonal Charcot-Marie-Tooth Disease. Hum Mutat, 37(5), 473-480. doi:10.1002/humu.22959

Horga, A., Laurà, M., Jaunmuktane, Z., Jerath, N. U., Gonzalez, M. A., Polke, J. M., . . Reilly, M. M. (2017). Genetic and clinical characteristics of NEFL-related Charcot-Marie-Tooth disease. J Neurol Neurosurg Psychiatry, 88(7), 575-585. doi:10.1136/jnnp-2016-315077

Houlden, H., Laura, M., Wavrant-De Vrièze, F., Blake, J., Wood, N., \& Reilly, M. M. (2008). Mutations in the HSP27 (HSPB1) gene cause dominant, recessive, and sporadic distal HMN/CMT type 2. Neurology, 71(21), 1660-1668. doi:10.1212/01.wnl.0000319696.14225.67

Høyer, H., Braathen, G. J., Busk $\varnothing$, L., Holla $\varnothing$, L., Svendsen, M., Hilmarsen, H. T., . . Russell, M. B. (2014). Genetic diagnosis of Charcot-Marie-Tooth disease in a population by next-generation sequencing. Biomed Res Int, 2014, 210401. doi:10.1155/2014/210401

Høyer, H., Braathen, G. J., Eek, A. K., Skjelbred, C. F., \& Russell, M. B. (2011). Charcot-Marie-Tooth caused by a copy number variation in myelin protein zero. Eur J Med Genet, 54(6), e580-583. doi:10.1016/j.ejmg.2011.06.006

lapadre, G., Morana, G., Vari, M. S., Pinto, F., Lanteri, P., Tessa, A., ... Verrotti, A. (2018). A novel homozygous MFN2 mutation associated with severe and atypical CMT2 phenotype. Eur J Paediatr Neurol, 22(3), 563-567. doi:10.1016/j.ejpn.2017.12.020 
James, P. A., Cader, M. Z., Muntoni, F., Childs, A. M., Crow, Y. J., \& Talbot, K. (2006). Severe childhood SMA and axonal CMT due to anticodon binding domain mutations in the GARS gene. Neurology, 67(9), 1710-1712. doi:10.1212/01.wnl.0000242619.52335.bc

Jin, S., Wang, W., Wang, R., Lv, H., Zhang, W., Wang, Z., . . Yuan, Y. (2015). INF2 mutations associated with dominant inherited intermediate Charcot-Marie-Tooth neuropathy with focal segmental glomerulosclerosis in two Chinese patients. Clin Neuropathol, 34(5), 275-281. doi:10.5414/NP300835

Jordanova, A., Irobi, J., Thomas, F. P., Van Dijck, P., Meerschaert, K., Dewil, M., . . Timmerman, V. (2006). Disrupted function and axonal distribution of mutant tyrosyl-tRNA synthetase in dominant intermediate Charcot-Marie-Tooth neuropathy. Nat Genet, 38(2), 197-202. doi:10.1038/ng1727

Kabzińska, D., Saifi, G. M., Drac, H., Rowińska-Marcińska, K., Hausmanowa-Petrusewicz, I., Kochański, A., \& Lupski, J. R. (2007). Charcot-Marie-Tooth disease type 4C4 caused by a novel Pro153Leu substitution in the GDAP1 gene. Acta Myol, 26(2), 108-111.

Kanwal, S., \& Perveen, S. (2019). Association of SNP in JPH1 gene with severity of disease in Charcot Marie Tooth 2K patients. J Pak Med Assoc, 69(2), 241-243.

Kijima, K., Numakura, C., Izumino, H., Umetsu, K., Nezu, A., Shiiki, T., ... Hayasaka, K. (2005). Mitochondrial GTPase mitofusin 2 mutation in Charcot-Marie-Tooth neuropathy type 2A. Hum Genet, 116(1-2), 23-27. doi:10.1007/s00439-004-1199-2

Kim, H. J., Hong, Y. B., Park, J. M., Choi, Y. R., Kim, Y. J., Yoon, B. R., . . Choi, B. O. (2013). Mutations in the PLEKHG5 gene is relevant with autosomal recessive intermediate Charcot-Marie-Tooth disease. Orphanet J Rare Dis, 8, 104. doi:10.1186/1750-1172-8-104

Kim, S. J., Nam, S. H., Kanwal, S., Nam, D. E., Yoo, D. H., Chae, J. H., . . Choi, B. O. (2018). BAG3 mutation in a patient with atypical phenotypes of myofibrillar myopathy and Charcot-Marie-Tooth disease. Genes Genomics, 40(12), 1269-1277. doi:10.1007/s13258-018-0721-1

Kleffner, I., Schirmacher, A., Gess, B., Boentert, M., \& Young, P. (2010). Four novel mutations of the myelin protein zero gene presenting as a mild and late-onset polyneuropathy. J Neurol, 257(11), 1864-1868. doi:10.1007/s00415-010-5624-2

Klein, C. J., Cunningham, J. M., Atkinson, E. J., Schaid, D. J., Hebbring, S. J., Anderson, S. A., . . Dyck, P. J. (2003). The gene for HMSN2C maps to 12q23-24: a region of neuromuscular disorders. Neurology, 60(7), 1151-1156. doi:10.1212/01.wnl.0000055900.30217.ea

Kochański, A., Kabzińska, D., Drac, H., Ryniewicz, B., Rowińska-Marcińska, K., \& HausmanowaPetrusewicz, I. (2004). Early onset Charcot-Marie-Tooth type 1B disease caused by a novel Leu190fs mutation in the myelin protein zero gene. Eur J Paediatr Neurol, 8(4), 221-224. doi:10.1016/j.ejpn.2004.04.001

Korobova, F., Gauvin, T. J., \& Higgs, H. N. (2014). A role for myosin II in mammalian mitochondrial fission. Curr Biol, 24(4), 409-414. doi:10.1016/j.cub.2013.12.032

Korobova, F., Ramabhadran, V., \& Higgs, H. N. (2013). An actin-dependent step in mitochondrial fission mediated by the ER-associated formin INF2. Science, 339(6118), 464-467. doi:10.1126/science.1228360

Kotruchow, K., Kabzińska, D., Hausmanowa-Petrusewicz, I., \& Kochański, A. (2013). A late-onset and mild form of Charcot-Marie-Tooth disease type 2 caused by a novel splice-site mutation within the Mitofusin-2 gene. Acta Myol, 32(3), 166-169.

Kurihara, S., Adachi, Y., Imai, C., Araki, H., Hattori, N., Numakura, C., . . Nakashima, K. (2004). CharcotMarie-Tooth families in Japan with MPZ Thr124Met mutation. J Neurol Neurosurg Psychiatry, 75(10), 1492-1494. doi:10.1136/jnnp.2003.020107 
Lagueny, A., Latour, P., Vital, A., Rajabally, Y., Le Masson, G., Ferrer, X., ... Vandenberghe, A. (1999). Peripheral myelin modification in CMT1B correlates with MPZ gene mutations. Neuromuscul Disord, 9(6-7), 361-367. doi:10.1016/s0960-8966(99)00031-0

Larrea, D., Pera, M., Gonnelli, A., Quintana-Cabrera, R., Akman, H. O., Guardia-Laguarta, C., .. . Giacomello, M. (2019). MFN2 mutations in Charcot-Marie-Tooth disease alter mitochondriaassociated ER membrane function but do not impair bioenergetics. Hum Mol Genet, 28(11), 1782-1800. doi:10.1093/hmg/ddz008

Laššuthová, P., Brožková, D., Krůtová, M., Neupauerová, J., Haberlová, J., Mazanec, R., . . Seeman, P. (2015). Mutations in HINT1 are one of the most frequent causes of hereditary neuropathy among Czech patients and neuromyotonia is rather an underdiagnosed symptom. Neurogenetics, 16(1), 43-54. doi:10.1007/s10048-014-0427-8

Lassuthova, P., Rebelo, A. P., Ravenscroft, G., Lamont, P. J., Davis, M. R., Manganelli, F., . . Zuchner, S. (2018). Mutations in ATP1A1 Cause Dominant Charcot-Marie-Tooth Type 2. Am J Hum Genet, 102(3), 505-514. doi:10.1016/j.ajhg.2018.01.023

Latour, P., Blanquet, F., Nelis, E., Bonnebouche, C., Chapon, F., Diraison, P., . . et al. (1995). Mutations in the myelin protein zero gene associated with Charcot-Marie-Tooth disease type 1B. Hum Mutat, 6(1), 50-54. doi:10.1002/humu.1380060110

Laurà, M., Milani, M., Morbin, M., Moggio, M., Ripolone, M., Jann, S., . . Pareyson, D. (2007). Rapid progression of late onset axonal Charcot-Marie-Tooth disease associated with a novel MPZ mutation in the extracellular domain. J Neurol Neurosurg Psychiatry, 78(11), 1263-1266. doi:10.1136/jnnp.2006.112276

Lawson, V. H., Graham, B. V., \& Flanigan, K. M. (2005). Clinical and electrophysiologic features of CMT2A with mutations in the mitofusin 2 gene. Neurology, 65(2), 197-204. doi:10.1212/01.wnl.0000168898.76071.70

Leal, A., Berghoff, C., Berghoff, M., Rojas-Araya, M., Carolina, O., Heuss, D., . . Rautenstrauss, B. (2014). A Costa Rican family affected with Charcot-Marie-Tooth disease due to the myelin protein zero (MPZ) p.Thr124Met mutation shares the Belgian haplotype. Rev Biol Trop, 62(4), 1285-1293. doi:10.15517/rbt.v62i4.13473

Leal, A., Bogantes-Ledezma, S., Ekici, A. B., Uebe, S., Thiel, C. T., Sticht, H., . . Reis, A. (2018). The polynucleotide kinase 3'-phosphatase gene (PNKP) is involved in Charcot-Marie-Tooth disease (CMT2B2) previously related to MED25. Neurogenetics, 19(4), 215-225. doi:10.1007/s10048018-0555-7

Leal, A., Huehne, K., Bauer, F., Sticht, H., Berger, P., Suter, U., . . Rautenstrauss, B. (2009). Identification of the variant Ala335Val of MED25 as responsible for CMT2B2: molecular data, functional studies of the $\mathrm{SH} 3$ recognition motif and correlation between wild-type MED25 and PMP22 RNA levels in CMT1A animal models. Neurogenetics, 10(4), 275-287. doi:10.1007/s10048-009-0183-3

Lee, Y. C., Yu, C. T., Lin, K. P., Chang, M. H., Hsu, S. L., Liu, Y. F., . . Soong, B. W. (2008). MPZ mutation G123S characterization: evidence for a complex pathogenesis in CMT disease. Neurology, 70(4), 273-277. doi:10.1212/01.wnl.0000296828.66915.bf

Liang, J. J., Grogan, M., Ackerman, M. J., \& Goodsell, K. (2016). LMNA-Mediated Arrhythmogenic Right Ventricular Cardiomyopathy and Charcot-Marie-Tooth Type 2B1: A Patient-Discovered Unifying Diagnosis. J Cardiovasc Electrophysiol, 27(7), 868-871. doi:10.1111/jce.12984

Liu, X. M., Tang, B. S., Zhao, G. H., Xia, K., Zhang, F. F., Pan, Q., . . Jiang, H. (2005). [Mutation analysis of small heat shock protein 27 gene in Chinese patients with Charcot-Marie-Tooth disease]. Zhonghua Yi Xue Yi Chuan Xue Za Zhi, 22(5), 510-513.

Loiseau, D., Chevrollier, A., Verny, C., Guillet, V., Gueguen, N., Pou de Crescenzo, M. A., . . Reynier, P. (2007). Mitochondrial coupling defect in Charcot-Marie-Tooth type 2A disease. Ann Neurol, 61(4), 315-323. doi:10.1002/ana.21086 
Luo, W., Tang, B., Zhao, G., Li, Q., Xiao, J., Yang, Q., \& Xia, J. (2003). [Mutation analysis of neurofilamentlight gene in Chinese Charcot-Marie-Tooth disease]. Zhonghua Yi Xue Yi Chuan Xue Za Zhi, 20(2), 169-170.

Lupo, V., García-García, F., Sancho, P., Tello, C., García-Romero, M., Villarreal, L., . . Espinós, C. (2016). Assessment of Targeted Next-Generation Sequencing as a Tool for the Diagnosis of CharcotMarie-Tooth Disease and Hereditary Motor Neuropathy. J Mol Diagn, 18(2), 225-234. doi:10.1016/j.jmoldx.2015.10.005

Lupski, J. R. (2000). Axonal Charcot-Marie-Tooth disease and the neurofilament light gene (NF-L). Am J Hum Genet, 67(1), 8-10. doi:10.1086/302986

Lv, H., Wang, L., Li, W., Qiao, X., Li, Y., Wang, Z., \& Yuan, Y. (2013). Mitofusin 2 gene mutation causing early-onset CMT2A with different progressive courses. Clin Neuropathol, 32(1), 16-23. doi:10.5414/np300464

Lv, H., Wang, L., Zhang, W., Wang, Z., Zuo, Y., Liu, J., \& Yuan, Y. (2015). A cohort study of Han Chinese MFN2-related Charcot-Marie-Tooth 2A. J Neurol Sci, 358(1-2), 153-157. doi:10.1016/j.jns.2015.08.1528

Mademan, I., Deconinck, T., Dinopoulos, A., Voit, T., Schara, U., Devriendt, K., . . Baets, J. (2013). De novo INF2 mutations expand the genetic spectrum of hereditary neuropathy with glomerulopathy. Neurology, 81(22), 1953-1958. doi:10.1212/01.wnl.0000436615.58705.c9

Maeda, M. H., Mitsui, J., Soong, B. W., Takahashi, Y., Ishiura, H., Hayashi, S., . . Tsuji, S. (2012). Increased gene dosage of myelin protein zero causes Charcot-Marie-Tooth disease. Ann Neurol, 71(1), 84-92. doi:10.1002/ana.22658

Mai, P. T., Le, D. T., Nguyen, T. T., Le Gia, H. L., Nguyen Le, T. H., Le, M., \& Do, D. M. (2019). Novel GDAP1 Mutation in a Vietnamese Family with Charcot-Marie-Tooth Disease. Biomed Res Int, 2019, 7132494. doi:10.1155/2019/7132494

Manganelli, F., Nolano, M., Pisciotta, C., Provitera, V., Fabrizi, G. M., Cavallaro, T., .. Santoro, L. (2015). Charcot-Marie-Tooth disease: New insights from skin biopsy. Neurology, 85(14), 1202-1208. doi:10.1212/wnl.0000000000001993

Manor, U., Bartholomew, S., Golani, G., Christenson, E., Kozlov, M., Higgs, H., . . Lippincott-Schwartz, J. (2015). A mitochondria-anchored isoform of the actin-nucleating spire protein regulates mitochondrial division. Elife, 4. doi:10.7554/eLife.08828

Martí, S., León, M., Orellana, C., Prieto, J., Ponsoda, X., López-García, C., . . Torres, J. (2017). Generation of a disease-specific iPS cell line derived from a patient with Charcot-Marie-Tooth type $2 \mathrm{~K}$ lacking functional GDAP1 gene. Stem Cell Res, 18, 1-4. doi:10.1016/j.scr.2016.11.017

Martin, A. M., Maradei, S. J., \& Velasco, H. M. (2015). Charcot Marie Tooth disease (CMT4A) due to GDAP1 mutation: report of a Colombian family. Colomb Med (Cali), 46(4), 194-198.

Marttila, M., Rautenstrauss, B., Huehne, K., Laitinen, V., Majamaa, K., \& Kärppä, M. (2012). A novel mutation of myelin protein zero associated with late-onset predominantly axonal CharcotMarie-Tooth disease. J Neurol, 259(8), 1585-1589. doi:10.1007/s00415-011-6382-5

Masingue, M., Perrot, J., Carlier, R. Y., Piguet-Lacroix, G., Latour, P., \& Stojkovic, T. (2018). WES homozygosity mapping in a recessive form of Charcot-Marie-Tooth neuropathy reveals intronic GDAP1 variant leading to a premature stop codon. Neurogenetics, 19(2), 67-76. doi:10.1007/s10048-018-0539-7

Mazzeo, A., Muglia, M., Rodolico, C., Toscano, A., Patitucci, A., Quattrone, A., . . Vita, G. (2008). Charcot-Marie-Tooth disease type 1B: marked phenotypic variation of the Ser78Leu mutation in five Italian families. Acta Neurol Scand, 118(5), 328-332. doi:10.1111/j.1600-0404.2008.01021.x

McCorquodale, D. S., 3rd, Montenegro, G., Peguero, A., Carlson, N., Speziani, F., Price, J., ... Züchner, S. (2011). Mutation screening of mitofusin 2 in Charcot-Marie-Tooth disease type 2. J Neurol, 258(7), 1234-1239. doi:10.1007/s00415-011-5910-7 
McLaughlin, H. M., Sakaguchi, R., Giblin, W., Wilson, T. E., Biesecker, L., Lupski, J. R., . . Antonellis, A. (2012). A recurrent loss-of-function alanyl-tRNA synthetase (AARS) mutation in patients with Charcot-Marie-Tooth disease type 2N (CMT2N). Hum Mutat, 33(1), 244-253. doi:10.1002/humu.21635

McLaughlin, H. M., Sakaguchi, R., Liu, C., Igarashi, T., Pehlivan, D., Chu, K., ... Antonellis, A. (2010). Compound heterozygosity for loss-of-function lysyl-tRNA synthetase mutations in a patient with peripheral neuropathy. Am J Hum Genet, 87(4), 560-566. doi:10.1016/j.ajhg.2010.09.008

Meggouh, F., Bienfait, H. M., Weterman, M. A., de Visser, M., \& Baas, F. (2006). Charcot-Marie-Tooth disease due to a de novo mutation of the RAB7 gene. Neurology, 67(8), 1476-1478. doi:10.1212/01.wnl.0000240068.21499.f5

Milley, G. M., Varga, E. T., Grosz, Z., Nemes, C., Arányi, Z., Boczán, J., . . . Gál, A. (2018). Genotypic and phenotypic spectrum of the most common causative genes of Charcot-Marie-Tooth disease in Hungarian patients. Neuromuscul Disord, 28(1), 38-43. doi:10.1016/j.nmd.2017.08.007

Miltenberger-Miltenyi, G., Janecke, A. R., Wanschitz, J. V., Timmerman, V., Windpassinger, C., AuerGrumbach, M., \& Löscher, W. N. (2007). Clinical and electrophysiological features in CharcotMarie-Tooth disease with mutations in the NEFL gene. Arch Neurol, 64(7), 966-970. doi:10.1001/archneur.64.7.966

Misgeld, T., \& Schwarz, T. L. (2017). Mitostasis in Neurons: Maintaining Mitochondria in an Extended Cellular Architecture. Neuron, 96(3), 651-666. doi:10.1016/j.neuron.2017.09.055

Miura, S., Morikawa, T., Fujioka, R., Noda, K., Kosaka, K., Taniwaki, T., \& Shibata, H. (2017). A novel missense variant (GIn220Arg) of GNB4 encoding guanine nucleotide-binding protein, subunit beta-4 in a Japanese family with autosomal dominant motor and sensory neuropathy. Eur J Med Genet, 60(9), 474-478. doi:10.1016/j.ejmg.2017.06.006

Montecchiani, C., Pedace, L., Lo Giudice, T., Casella, A., Mearini, M., Gaudiello, F., ... Orlacchio, A. (2016). ALS5/SPG11/KIAA1840 mutations cause autosomal recessive axonal Charcot-MarieTooth disease. Brain, 139(Pt 1), 73-85. doi:10.1093/brain/awv320

Morelli, K. H., Seburn, K. L., Schroeder, D. G., Spaulding, E. L., Dionne, L. A., Cox, G. A., \& Burgess, R. W. (2017). Severity of Demyelinating and Axonal Neuropathy Mouse Models Is Modified by Genes Affecting Structure and Function of Peripheral Nodes. Cell Rep, 18(13), 3178-3191. doi:10.1016/j.celrep.2017.03.009

Moroni, I., Morbin, M., Milani, M., Ciano, C., Bugiani, M., Pagliano, E., .. . Taroni, F. (2009). Novel mutations in the GDAP1 gene in patients affected with early-onset axonal Charcot-Marie-Tooth type 4A. Neuromuscul Disord, 19(7), 476-480. doi:10.1016/j.nmd.2009.04.014

Motley, W. W., Seburn, K. L., Nawaz, M. H., Miers, K. E., Cheng, J., Antonellis, A., . . Burgess, R. W. (2011). Charcot-Marie-Tooth-linked mutant GARS is toxic to peripheral neurons independent of wild-type GARS levels. PLoS Genet, 7(12), e1002399. doi:10.1371/journal.pgen.1002399

Muglia, M., Vazza, G., Patitucci, A., Milani, M., Pareyson, D., Taroni, F., . . Mostacciuolo, M. L. (2007). A novel founder mutation in the MFN2 gene associated with variable Charcot-Marie-Tooth type 2 phenotype in two families from Southern Italy. J Neurol Neurosurg Psychiatry, 78(11), 12861287. doi:10.1136/jnnp.2007.115774

Nakagawa, M., \& Takashima, H. (2003). [Molecular mechanisms of hereditary neuropathy: genotypephenotype correlation]. Rinsho Byori, 51(6), 536-543.

Nelis, E., Timmerman, V., De Jonghe, P., Vandenberghe, A., Pham-Dinh, D., Dautigny, A., . . . Van Broeckhoven, C. (1994). Rapid screening of myelin genes in CMT1 patients by SSCP analysis: identification of new mutations and polymorphisms in the P0 gene. Hum Genet, 94(6), 653-657. doi:10.1007/bf00206959 
Neupauerová, J., Grečmalová, D., Seeman, P., \& Laššuthová, P. (2016). Massively Parallel Sequencing Detected a Mutation in the MFN2 Gene Missed by Sanger Sequencing Due to a Primer Mismatch on an SNP Site. Ann Hum Genet, 80(3), 182-186. doi:10.1111/ahg.12151

Neusch, C., Senderek, J., Eggermann, T., Elolff, E., Bahr, M., \& Schneider-Gold, C. (2007). Mitofusin 2 gene mutation (R94Q) causing severe early-onset axonal polyneuropathy (CMT2A). Eur J Neurol, 14(5), 575-577. doi:10.1111/j.1468-1331.2006.01688.x

Nicholson, G., Kennerson, M., Brewer, M., Garbern, J., \& Shy, M. (2009). Genotypes \& sensory phenotypes in 2 new X-linked neuropathies (CMTX3 and dSMAX) and dominant CMT/HMN overlap syndromes. Adv Exp Med Biol, 652, 201-206. doi:10.1007/978-90-481-2813-6_13

Nicolaou, P., Cianchetti, C., Minaidou, A., Marrosu, G., Zamba-Papanicolaou, E., Middleton, L., \& Christodoulou, K. (2013). A novel LRSAM1 mutation is associated with autosomal dominant axonal Charcot-Marie-Tooth disease. Eur J Hum Genet, 21(2), 190-194. doi:10.1038/ejhg.2012.146

Ouvrier, R., \& Grew, S. (2010). Mechanisms of disease and clinical features of mutations of the gene for mitofusin 2: an important cause of hereditary peripheral neuropathy with striking clinical variability in children and adults. Dev Med Child Neurol, 52(4), 328-330. doi:10.1111/j.14698749.2010.03613.x

Pan, Z. N., Pan, M. H., Sun, M. H., Li, X. H., Zhang, Y., \& Sun, S. C. (2020). RAB7 GTPase regulates actin dynamics for DRP1-mediated mitochondria function and spindle migration in mouse oocyte meiosis. FASEB J, 34(7), 9615-9627. doi:10.1096/fj.201903013R

Park, H. J., Kim, H. J., Hong, Y. B., Nam, S. H., Chung, K. W., \& Choi, B. O. (2014). A novel INF2 mutation in a Korean family with autosomal dominant intermediate Charcot-Marie-Tooth disease and focal segmental glomerulosclerosis. J Peripher Nerv Syst, 19(2), 175-179. doi:10.1111/jns5.12062

Park, S. Y., Kim, S. Y., Hong, Y. H., Cho, S. I., Seong, M. W., \& Park, S. S. (2012). A novel double mutation in cis in MFN2 causes Charcot-Marie-Tooth neuropathy type 2A. Neurogenetics, 13(3), 275-280. doi:10.1007/s10048-012-0327-8

Pathak, D., Sepp, K. J., \& Hollenbeck, P. J. (2010). Evidence that myosin activity opposes microtubulebased axonal transport of mitochondria. J Neurosci, 30(26), 8984-8992. doi:10.1523/jneurosci.1621-10.2010

Pehlivan, D., Coban Akdemir, Z., Karaca, E., Bayram, Y., Jhangiani, S., Yildiz, E. P., . . Harel, T. (2015). Exome sequencing reveals homozygous TRIM2 mutation in a patient with early onset CMT and bilateral vocal cord paralysis. Hum Genet, 134(6), 671-673. doi:10.1007/s00439-015-1548-3

Pham-Dinh, D., Fourbil, Y., Blanquet, F., Mattéi, M. G., Roeckel, N., Latour, P., . . Dautigny, A. (1993). The major peripheral myelin protein zero gene: structure and localization in the cluster of Fc gamma receptor genes on human chromosome 1q21.3-q23. Hum Mol Genet, 2(12), 2051-2054. doi:10.1093/hmg/2.12.2051

Pitceathly, R. D., Murphy, S. M., Cottenie, E., Chalasani, A., Sweeney, M. G., Woodward, C., .. Hanna, M. G. (2012). Genetic dysfunction of MT-ATP6 causes axonal Charcot-Marie-Tooth disease. Neurology, 79(11), 1145-1154. doi:10.1212/WNL.0b013e3182698d8d

Pla-Martín, D., Calpena, E., Lupo, V., Márquez, C., Rivas, E., Sivera, R., . . Espinós, C. (2015). Junctophilin-1 is a modifier gene of GDAP1-related Charcot-Marie-Tooth disease. Hum Mol Genet, 24(1), 213-229. doi:10.1093/hmg/ddu440

Qin, L., Yang, C., Lü, T., Li, L., Zong, D., \& Wu, Y. (2019). [Analysis of GDAP1 gene mutation in a pedigree with autosomal dominant Charcot-Marie-Tooth disease]. Nan Fang Yi Ke Da Xue Xue Bao, 39(1), 63-68. doi:10.12122/j.issn.1673-4254.2019.01.10

Quattrini, A., Feltri, M. L., Previtali, S., Fasolini, M., Messing, A., \& Wrabetz, L. (1999). Peripheral nerve dysmyelination due to P0 glycoprotein overexpression is dose-dependent. Ann N Y Acad Sci, 883, 294-301. 
Quintero, O. A., DiVito, M. M., Adikes, R. C., Kortan, M. B., Case, L. B., Lier, A. J., . . Cheney, R. E. (2009). Human Myo19 is a novel myosin that associates with mitochondria. Curr Biol, 19(23), 20082013. doi:10.1016/j.cub.2009.10.026

Rangaraju, V., Lauterbach, M., \& Schuman, E. M. (2019). Spatially Stable Mitochondrial Compartments Fuel Local Translation during Plasticity. Cell, 176(1-2), 73-84.e15. doi:10.1016/j.cell.2018.12.013

Roa, B. B., Warner, L. E., Garcia, C. A., Russo, D., Lovelace, R., Chance, P. F., \& Lupski, J. R. (1996). Myelin protein zero (MPZ) gene mutations in nonduplication type 1 Charcot-Marie-Tooth disease. Hum Mutat, 7(1), 36-45. doi:10.1002/(sici)1098-1004(1996)7:1<36::Aid-humu5>3.0.Co;2-n

Rocha, A. G., Franco, A., Krezel, A. M., Rumsey, J. M., Alberti, J. M., Knight, W. C., . . Dorn, G. W., 2nd. (2018). MFN2 agonists reverse mitochondrial defects in preclinical models of Charcot-MarieTooth disease type 2A. Science, 360(6386), 336-341. doi:10.1126/science.aao1785

Rodriguez, P. Q., Lohkamp, B., Celsi, G., Mache, C. J., Auer-Grumbach, M., Wernerson, A., . . Patrakka, J. (2013). Novel INF2 mutation p. L77P in a family with glomerulopathy and Charcot-Marie-Tooth neuropathy. Pediatr Nephrol, 28(2), 339-343. doi:10.1007/s00467-012-2299-1

Rosberg, M. R., Alvarez, S., Krarup, C., \& Moldovan, M. (2013). Functional recovery of regenerating motor axons is delayed in mice heterozygously deficient for the myelin protein $\mathrm{P}(0)$ gene. Neurochem Res, 38(6), 1266-1277. doi:10.1007/s11064-013-1030-3

Rougeot, C., Chabrier, S., Camdessanche, J. P., Prieur, F., d'Anjou, M. C., \& Latour, P. (2008). Clinical, electrophysiological and genetic studies of two families with mutations in the GDAP1 gene. Neuropediatrics, 39(3), 184-187. doi:10.1055/s-0028-1085467

Rudnik-Schöneborn, S., Tölle, D., Senderek, J., Eggermann, K., Elbracht, M., Kornak, U., . . Zerres, K. (2016). Diagnostic algorithms in Charcot-Marie-Tooth neuropathies: experiences from a German genetic laboratory on the basis of 1206 index patients. Clin Genet, 89(1), 34-43. doi:10.1111/cge.12594

Rzepnikowska, W., \& Kochański, A. (2018). A role for the GDAP1 gene in the molecular pathogenesis of Charcot-Marie-Tooth disease. Acta Neurobiologiae Experimentalis, 78(1), 1-13. doi:10.21307/ane-2018-002

Sabet, A., Li, J., Ghandour, K., Pu, Q., Wu, X., Kamholz, J., . . . Cambi, F. (2006). Skin biopsies demonstrate MPZ splicing abnormalities in Charcot-Marie-Tooth neuropathy 1B. Neurology, 67(7), 11411146. doi:10.1212/01.wnl.0000238499.37764.b1

Saghira, C., Bis, D. M., Stanek, D., Strickland, A., Herrmann, D. N., Reilly, M. M., . . Züchner, S. (2018). Variant pathogenicity evaluation in the community-driven Inherited Neuropathy Variant Browser. Hum Mutat, 39(5), 635-642. doi:10.1002/humu.23412

Sahin-Calapoglu, N., Soyoz, M., Calapoglu, M., \& Ozcelik, N. (2009). Genetic study of demyelinating form of autosomal-recessive Charcot-Marie-tooth diseases in a Turkish family. Int J Neurosci, 119(8), 1179-1189. doi:10.1080/00207450902869906

Sahin-Calapoglu, N., Tan, M., Soyoz, M., Calapoglu, M., \& Ozcelik, N. (2009). Novel GDAP1 mutation in a Turkish family with CMT2K (CMT2K with novel GDAP1 mutation). Neuromolecular Med, 11(2), 106-113. doi:10.1007/s12017-009-8062-5

Sanmaneechai, O., Feely, S., Scherer, S. S., Herrmann, D. N., Burns, J., Muntoni, F., . . Shy, M. E. (2015). Genotype-phenotype characteristics and baseline natural history of heritable neuropathies caused by mutations in the MPZ gene. Brain, 138(Pt 11), 3180-3192. doi:10.1093/brain/awv241

Schiavon, C. R., Zhang, T., Zhao, B., Moore, A. S., Wales, P., Andrade, L. R., . . Manor, U. (2020). Actin chromobody imaging reveals sub-organellar actin dynamics. Nat Methods, 17(9), 917-921. doi:10.1038/s41592-020-0926-5

Senderek, J., Bergmann, C., Ramaekers, V. T., Nelis, E., Bernert, G., Makowski, A., . . Schröder, J. M. (2003). Mutations in the ganglioside-induced differentiation-associated protein-1 (GDAP1) gene 
in intermediate type autosomal recessive Charcot-Marie-Tooth neuropathy. Brain, 126(Pt 3), 642-649. doi:10.1093/brain/awg068

Senderek, J., Hermanns, B., Lehmann, U., Bergmann, C., Marx, G., Kabus, C., . . Schröder, J. M. (2000). Charcot-Marie-Tooth neuropathy type 2 and PO point mutations: two novel amino acid substitutions (Asp61Gly; Tyr119Cys) and a possible "hotspot" on Thr124Met. Brain Pathol, 10(2), 235-248. doi:10.1111/j.1750-3639.2000.tb00257.x

Shin, J. S., Chung, K. W., Cho, S. Y., Yun, J., Hwang, S. J., Kang, S. H., . . Choi, B. O. (2008). NEFL Pro22Arg mutation in Charcot-Marie-Tooth disease type 1. J Hum Genet, 53(10), 936-940. doi:10.1007/s10038-008-0333-8

Shy, M., Rebelo, A. P., Feely, S. M., Abreu, L. A., Tao, F., Swenson, A., . . Zuchner, S. (2018). Mutations in BAG3 cause adult-onset Charcot-Marie-Tooth disease. J Neurol Neurosurg Psychiatry, 89(3), 313315. doi:10.1136/jnnp-2017-315929

Silander, K., Meretoja, P., Nelis, E., Timmerman, V., Van Broeckhoven, C., Aula, P., \& Savontaus, M. L. (1996). A de novo duplication in 17p11.2 and a novel mutation in the Po gene in two DéjérineSottas syndrome patients. Hum Mutat, 8(4), 304-310. doi:10.1002/(sici)10981004(1996)8:4<304::Aid-humu2>3.0.Co;2-7

Skre, H. (1974). Genetic and clinical aspects of Charcot-Marie-Tooth's disease. Clin Genet, 6(2), 98-118. Retrieved from https://onlinelibrary.wiley.com/doi/abs/10.1111/j.13990004.1974.tb00638.x?sid=nlm\%3Apubmed

Solla, P., Vannelli, A., Bolino, A., Marrosu, G., Coviello, S., Murru, M. R., . . Marrosu, M. G. (2010). Heat shock protein 27 R127W mutation: evidence of a continuum between axonal Charcot-MarieTooth and distal hereditary motor neuropathy. J Neurol Neurosurg Psychiatry, 81(9), 958-962. doi:10.1136/jnnp.2009.181636

Soong, B. W., Huang, Y. H., Tsai, P. C., Huang, C. C., Pan, H. C., Lu, Y. C., . . Lee, Y. C. (2013). Exome sequencing identifies GNB4 mutations as a cause of dominant intermediate Charcot-MarieTooth disease. Am J Hum Genet, 92(3), 422-430. doi:10.1016/j.ajhg.2013.01.014

Speevak, M. D., \& Farrell, S. A. (2013). Charcot-Marie-Tooth 1B caused by expansion of a familial myelin protein zero (MPZ) gene duplication. Eur J Med Genet, 56(10), 566-569. doi:10.1016/j.ejmg.2013.06.004

Sun, S. C., Ma, D., Li, M. Y., Zhang, R. X., Huang, C., Huang, H. J., . . Xu, X. M. (2019). Mutations in C1orf194, encoding a calcium regulator, cause dominant Charcot-Marie-Tooth disease. Brain, 142(8), 2215-2229. doi:10.1093/brain/awz151

Sun, Y., Hu, G., Luo, J., Fang, D., Yu, Y., Wang, X., ... Qiu, W. (2017). Mutations in methionyl-tRNA synthetase gene in a Chinese family with interstitial lung and liver disease, postnatal growth failure and anemia. J Hum Genet, 62(6), 647-651. doi:10.1038/jhg.2017.10

Tamiya, G., Makino, S., Hayashi, M., Abe, A., Numakura, C., Ueki, M., . . Hayasaka, K. (2014). A mutation of COX6A1 causes a recessive axonal or mixed form of Charcot-Marie-Tooth disease. Am J Hum Genet, 95(3), 294-300. doi:10.1016/j.ajhg.2014.07.013

Tan, C. A., Rabideau, M., Blevins, A., Westbrook, M. J., Ekstein, T., Nykamp, K., . . Demmer, L. (2016). Autosomal recessive MFN2-related Charcot-Marie-Tooth disease with diaphragmatic weakness: Case report and literature review. Am J Med Genet A, 170(6), 1580-1584. doi:10.1002/ajmg.a.37611

Tang, B., Liu, X., Zhao, G., Luo, W., Xia, K., Pan, Q., . . Jiang, H. (2005). Mutation analysis of the small heat shock protein 27 gene in chinese patients with Charcot-Marie-Tooth disease. Arch Neurol, 62(8), 1201-1207. doi:10.1001/archneur.62.8.1201

Tazir, M., Bellatache, M., Nouioua, S., \& Vallat, J. M. (2013). Autosomal recessive Charcot-Marie-Tooth disease: from genes to phenotypes. J Peripher Nerv Syst, 18(2), 113-129. doi:10.1111/jns5.12026 
Tétreault, M., Gonzalez, M., Dicaire, M. J., Allard, P., Gehring, K., Leblanc, D., . . Brais, B. (2015). Adultonset painful axonal polyneuropathy caused by a dominant NAGLU mutation. Brain, 138(Pt 6), 1477-1483. doi:10.1093/brain/awv074

Tokuda, N., Noto, Y., Kitani-Morii, F., Hamano, A., Kasai, T., Shiga, K., . . Mizuno, T. (2015). Parasympathetic Dominant Autonomic Dysfunction in Charcot-Marie-Tooth Disease Type 2J with the MPZ Thr124Met Mutation. Intern Med, 54(15), 1919-1922. doi:10.2169/internalmedicine.54.4259

Vallat, J. M., Mathis, S., \& Funalot, B. (2013). The various Charcot-Marie-Tooth diseases. Curr Opin Neurol, 26(5), 473-480. doi:10.1097/WCO.0b013e328364c04b

Vallat, J. M., Ouvrier, R. A., Pollard, J. D., Magdelaine, C., Zhu, D., Nicholson, G. A., . . Funalot, B. (2008). Histopathological findings in hereditary motor and sensory neuropathy of axonal type with onset in early childhood associated with mitofusin 2 mutations. J Neuropathol Exp Neurol, 67(11), 1097-1102. doi:10.1097/NEN.0b013e31818b6cbc

Venkatesh, K., Mathew, A., \& Koushika, S. P. (2019). Role of actin in organelle trafficking in neurons. Cytoskeleton (Hoboken). doi:10.1002/cm.21580

Verhoeven, K., Claeys, K. G., Zuchner, S., Schroder, J. M., Weis, J., Ceuterick, C., . . . Timmerman, V. (2006). MFN2 mutation distribution and genotype/phenotype correlation in Charcot-MarieTooth type 2. Brain, 129(Pt 8), 2093-2102. doi:10.1093/brain/awl126

Verhoeven, K., Claeys, K. G., Züchner, S., Schröder, J. M., Weis, J., Ceuterick, C., . . Timmerman, V. (2006). MFN2 mutation distribution and genotype/phenotype correlation in Charcot-MarieTooth type 2. Brain, 129(Pt 8), 2093-2102. doi:10.1093/brain/awl126

Vielhaber, S., Debska-Vielhaber, G., Peeva, V., Schoeler, S., Kudin, A. P., Minin, I., . . Kunz, W. S. (2013). Mitofusin 2 mutations affect mitochondrial function by mitochondrial DNA depletion. Acta Neuropathol, 125(2), 245-256. doi:10.1007/s00401-012-1036-y

Vital, A., Latour, P., Sole, G., Ferrer, X., Rouanet, M., Tison, F., . . Goizet, C. (2012). A French family with Charcot-Marie-Tooth disease related to simultaneous heterozygous MFN2 and GDAP1 mutations. Neuromuscul Disord, 22(8), 735-741. doi:10.1016/j.nmd.2012.04.001

Wagner, J. D., Huang, L., Tetreault, M., Majewski, J., Boycott, K. M., Bulman, D. E., . . McMillan, H. J. (2015). Autosomal recessive axonal polyneuropathy in a sibling pair due to a novel homozygous mutation in IGHMBP2. Neuromuscul Disord, 25(10), 794-799. doi:10.1016/j.nmd.2015.07.017

Wang, R., He, J., Li, J. J., Ni, W., Wu, Z. Y., Chen, W. J., \& Wang, Y. (2015). Clinical and genetic spectra in a series of Chinese patients with Charcot-Marie-Tooth disease. Clin Chim Acta, 451(Pt B), 263-270. doi:10.1016/j.cca.2015.10.007

Weedon, M. N., Hastings, R., Caswell, R., Xie, W., Paszkiewicz, K., Antoniadi, T., ... Ellard, S. (2011). Exome sequencing identifies a DYNC1H1 mutation in a large pedigree with dominant axonal Charcot-Marie-Tooth disease. Am J Hum Genet, 89(2), 308-312. doi:10.1016/j.ajhg.2011.07.002

Weeks, S. D., Muranova, L. K., Heirbaut, M., Beelen, S., Strelkov, S. V., \& Gusev, N. B. (2018). Characterization of human small heat shock protein HSPB1 $\alpha$-crystallin domain localized mutants associated with hereditary motor neuron diseases. Sci Rep, 8(1), 688. doi:10.1038/s41598-017-18874-x

Werheid, F., Azzedine, H., Zwerenz, E., Bozkurt, A., Moeller, M. J., Lin, L., . . Claeys, K. G. (2016). Underestimated associated features in CMT neuropathies: clinical indicators for the causative gene? Brain Behav, 6(4), e00451. doi:10.1002/brb3.451

Weterman, M. A. J., Kuo, M., Kenter, S. B., Gordillo, S., Karjosukarso, D. W., Takase, R., . . Baas, F. (2018). Hypermorphic and hypomorphic AARS alleles in patients with CMT2N expand clinical and molecular heterogeneities. Hum Mol Genet, 27(23), 4036-4050. doi:10.1093/hmg/ddy290

Wong, Y. C., Peng, W., \& Krainc, D. (2019). Lysosomal Regulation of Inter-mitochondrial Contact Fate and Motility in Charcot-Marie-Tooth Type 2. Dev Cell. doi:10.1016/j.devcel.2019.05.033 
Wong, Y. C., Ysselstein, D., \& Krainc, D. (2018). Mitochondria-lysosome contacts regulate mitochondrial fission via RAB7 GTP hydrolysis. Nature, 554(7692), 382-386. doi:10.1038/nature25486

Xie, W., Nangle, L. A., Zhang, W., Schimmel, P., \& Yang, X. L. (2007). Long-range structural effects of a Charcot-Marie-Tooth disease-causing mutation in human glycyl-tRNA synthetase. Proc Natl Acad Sci U S A, 104(24), 9976-9981. doi:10.1073/pnas.0703908104

Xie, W., Schimmel, P., \& Yang, X. L. (2006). Crystallization and preliminary X-ray analysis of a native human tRNA synthetase whose allelic variants are associated with Charcot-Marie-Tooth disease. Acta Crystallogr Sect F Struct Biol Cryst Commun, 62(Pt 12), 1243-1246. doi:10.1107/s1744309106046434

Xie, Y., Li, X., Liu, L., Hu, Z., Huang, S., Zhan, Y., . . Zhang, R. (2016). MFN2-related genetic and clinical features in a cohort of Chinese CMT2 patients. J Peripher Nerv Syst, 21(1), 38-44. doi:10.1111/jns.12159

Xin, B., Puffenberger, E., Nye, L., Wiznitzer, M., \& Wang, H. (2008). A novel mutation in the GDAP1 gene is associated with autosomal recessive Charcot-Marie-Tooth disease in an Amish family. Clin Genet, 74(3), 274-278. doi:10.1111/j.1399-0004.2008.01018.x

Xu, J. L., Zhang, Y., Zhao, C. Y., Jiang, P. F., Yuan, Z. F., Yu, Y. L., . . Gao, F. (2019). [A genotyping study of 13 cases of early-onset Charcot-Marie-Tooth disease]. Zhongguo Dang Dai Er Ke Za Zhi, 21(7), 670-675. doi:10.7499/j.issn.1008-8830.2019.07.010

Yang, C., \& Svitkina, T. M. (2019). Ultrastructure and dynamics of the actin-myosin II cytoskeleton during mitochondrial fission. Nat Cell Biol, 21(5), 603-613. doi:10.1038/s41556-019-0313-6

Yoshimura, A., Yuan, J. H., Hashiguchi, A., Hiramatsu, Y., Ando, M., Higuchi, Y., . . Takashima, H. (2017). Clinical and mutational spectrum of Japanese patients with Charcot-Marie-Tooth disease caused by GDAP1 variants. Clin Genet, 92(3), 274-280. doi:10.1111/cge.13002

Yu, S. B., \& Pekkurnaz, G. (2018). Mechanisms Orchestrating Mitochondrial Dynamics for Energy Homeostasis. J Mol Biol, 430(21), 3922-3941. doi:10.1016/j.jmb.2018.07.027

Yuan, J. H., Hashiguchi, A., Yoshimura, A., Yaguchi, H., Tsuzaki, K., Ikeda, A., . . Takashima, H. (2017). Clinical diversity caused by novel IGHMBP2 variants. J Hum Genet, 62(6), 599-604. doi:10.1038/jhg.2017.15

Zhang, R. X., Guo, P., Ren, Z. J., Zhao, G. H., Liu, S. M., Liu, T., .. . Tang, B. S. (2010). [Mutation analysis of LITAF, RAB7, LMNA and MTMR2 genes in Chinese Charcot-Marie-Tooth disease.]. Yi Chuan, 32(8), 817-823. doi:10.3724/sp.j.1005.2010.00817

Zhang, R. X., Tang, B. S., Zi, X. H., Luo, W., Xia, K., Pan, Q., . . Li, X. B. (2004). [Mutation analysis of ganglioside-induced differentiation associated protein-1 gene in Chinese Charcot-Marie-Tooth disease]. Zhonghua Yi Xue Yi Chuan Xue Za Zhi, 21(3), 207-210.

Zhao, Z., Hashiguchi, A., Hu, J., Sakiyama, Y., Okamoto, Y., Tokunaga, S., . . Takashima, H. (2012). AlanyltRNA synthetase mutation in a family with dominant distal hereditary motor neuropathy. Neurology, 78(21), 1644-1649. doi:10.1212/WNL.0b013e3182574f8f

Zhao, Z. H., Chen, Z. T., Zhou, R. L., \& Wang, Y. Z. (2019). A Chinese pedigree with a novel mutation in GJB1 gene and a rare variation in DHTKD1 gene for diverse Charcot-Marie-Tooth diseases. Mol Med Rep, 19(5), 4484-4490. doi:10.3892/mmr.2019.10058

Zimoń, M., Baets, J., Fabrizi, G. M., Jaakkola, E., Kabzińska, D., Pilch, J., . . Jordanova, A. (2011). Dominant GDAP1 mutations cause predominantly mild CMT phenotypes. Neurology, 77(6), 540548. doi:10.1212/WNL.0b013e318228fc70

Zimoń, M., Battaloğlu, E., Parman, Y., Erdem, S., Baets, J., De Vriendt, E., . . Jordanova, A. (2015). Unraveling the genetic landscape of autosomal recessive Charcot-Marie-Tooth neuropathies using a homozygosity mapping approach. Neurogenetics, 16(1), 33-42. doi:10.1007/s10048-0140422-0 
Zuchner, S., De Jonghe, P., Jordanova, A., Claeys, K. G., Guergueltcheva, V., Cherninkova, S., . . Vance, J. M. (2006). Axonal neuropathy with optic atrophy is caused by mutations in mitofusin 2 . Ann Neurol, 59(2), 276-281. doi:10.1002/ana.20797 